\title{
Molecular gas in NUclei of GAlaxies (NUGA) XIII. The interacting Seyfert 2/LINER galaxy NGC 5953^
}

\author{
V. Casasola ${ }^{1,2}$, L. K. Hunt ${ }^{1}$, F. Combes ${ }^{3}$, S. García-Burillo ${ }^{4}$, F. Boone ${ }^{3}$, A. Eckart ${ }^{5}$, R. Neri ${ }^{6}$, and E. Schinnerer ${ }^{7}$ \\ 1 INAF - Osservatorio Astrofisico di Arcetri, Largo E. Fermi 5, 50125 Firenze, Italy \\ e-mail: casasola@arcetri.astro.it \\ 2 INAF - Istituto di Radioastronomia, via P. Gobetti 101, 40129 Bologna, Italy \\ Observatoire de Paris, LERMA, 61 Av. de l'Observatoire, 75014 Paris, France \\ 4 Observatorio Astronómico Nacional (OAN) - Observatorio de Madrid, C/ Alfonso XII, 3, 28014 Madrid, Spain \\ 5 I. Physikalisches Institut, Universität zu Köln, Zülpicherstrasse 77, 50937 Köln, Germany \\ ${ }^{6}$ IRAM - Institut de Radio Astronomie Millimétrique, 300 rue de la Piscine, 38406 St. Martin d'Hères, France \\ 7 Max-Planck-Institut für Astronomie, Königstuhl 17, 69117 Heidelberg, Germany
}

Received 3 August 2009 / Accepted 7 November 2009

ABSTRACT

\begin{abstract}
We present ${ }^{12} \mathrm{CO}(1-0)$ and ${ }^{12} \mathrm{CO}(2-1)$ maps of the interacting Seyfert 2/LINER galaxy NGC 5953 obtained with the IRAM interferometer at resolutions of $2^{\prime \prime} 1 \times 1^{\prime \prime} .4$ and $1^{\prime \prime} .1 \times 0$ !'7, respectively. We also present single-dish IRAM $30 \mathrm{~m}$ observations of the central region of $\mathrm{NGC} 5953$ for the ${ }^{12} \mathrm{CO}(1-0),{ }^{12} \mathrm{CO}(2-1)$, and $\mathrm{HCN}(1-0)$ transitions at resolutions of $22^{\prime \prime}, 12^{\prime \prime}$, and 29", respectively. The CO emission is distributed over a disk of diameter $\sim 16^{\prime \prime}(\sim 2.2 \mathrm{kpc})$, within which are several, randomly distributed peaks. The strongest peak does not coincide with the nucleus, but is instead offset from the center, $\sim 2-3^{\prime \prime}(\sim 340 \mathrm{pc})$ toward the west/southwest. The kinematics of the molecular component are quite regular, as is typical of a rotating disk. We also compared the ${ }^{12} \mathrm{CO}$ distribution of NGC 5953 with observations at other wavelengths in order to study correlations between different tracers of the interstellar medium. The HST/F606W WFPC2 images show flocculent spiral structures and an "S-shape" feature $\gtrsim 60$ pc in radius, possibly associated with a nuclear bar or with the radio jet. A two-dimensional bulge/disk decomposition of the $H$-band (HST/F160W) and $3.6 \mu \mathrm{m}$ (Spitzer/IRAC) images reveals a circumnuclear "ring" $\sim 10-14$ " in diameter, roughly coincident in size with the CO disk and with a star-forming ring previously identified in ionized gas. This ring is not present in the near-infrared (NIR) $J-K$ color image, nor is it present in the "dust-only" image constructed from the $8 \mu \mathrm{m}$ IRAC map. The implication is that the excess residual ring is stellar, with colors similar to the surrounding disk. We interpret this ring, visible in ionized gas, which appears as stars in the NIR, and with no sign of hot dust, as due to a red super giant population at least 10-15 Myr old. However, star formation is still ongoing in the disk and in the ring itself. Using NIR images, we computed the gravity torques exerted by the stellar potential on the gas. The torques are predominantly positive in both ${ }^{12} \mathrm{CO}(1-0)$ and ${ }^{12} \mathrm{CO}(2-1)$, suggesting that gas is not flowing into the center, and less than $5 \%$ of the gas angular momentum is exchanged in each rotation. This comes from the regular and almost axisymmetric total mass and gas distributions in the center of the galaxy. In NGC 5953, the AGN is apparently not being actively fueled in the current epoch.
\end{abstract}

Key words. galaxies: individual: NGC 5953 - galaxies: spiral - galaxies: active - galaxies: nuclei - galaxies: ISM galaxies: kinematics and dynamics

\section{Introduction}

Since molecular gas is the predominant phase of the interstellar medium (ISM) in the inner regions of spiral galaxies, CO lines represent an optimum tracer of nuclear gas dynamics, active galactic nuclei (AGN) fueling mechanisms, and their link with circumnuclear star formation. Although most galaxies host super massive black holes (SMBHs) and the gas accretion phenomenon is usually invoked to explain nuclear activity in galaxies, the nature of this activity is still not well known. The feeding of an AGN through accretion depends on an adequate supply of gas whose angular momentum has been reduced enough to enable inflow on the small spatial scales surrounding the $\mathrm{BH}$. Angular momentum must be removed from the disk gas (e.g.,

* Based on observations carried out with the IRAM Plateau de Bure Interferometer. IRAM is supported by the INSU/CNRS (France), MPG (Germany), and IGN (Spain).
Jogee 2006, and references therein), a process that can be accomplished through non-axisymmetric perturbations of internal or external origin. In the first case, they arise from disk instabilities and, in the second, from galaxy collisions, mergers, and mass accretion (Heckman et al. 1986). Either way, they usually manifest themselves as density waves, such as large-scale spirals or as bars and their gravity torques (e.g., Sakamoto et al. 1999; Combes 2001), or as more localized phenomena, including nested nuclear bars (e.g., Friedli \& Martinet 1993), lopsidedness or $m=1$ perturbations (e.g., Shu et al. 1990; García-Burillo et al. 2000), or warped nuclear disks (e.g., Schinnerer et al. 2000a,b).

To better understand the mechanisms for gas fueling of AGN, we started a high-resolution and high-sensitivity CO survey of nearby active galaxies at the IRAM Plateau de Bure Interferometer (PdBI), the NUclei of GAlaxies (NUGA) project (García-Burillo et al. 2003). The galaxies of the NUGA sample already studied show surprising results. In fact, there is 
no unique circumnuclear molecular gas feature linked with nuclear activity, but instead a variety of molecular gas morphologies which characterize the inner kpc of active galaxies. These morphologies include one- and two-armed instabilities (GarcíaBurillo et al. 2003), well-ordered rings and nuclear spirals (Combes et al. 2004; Casasola et al. 2008), circumnuclear asymmetries (Krips et al. 2005) and large-scale bars (Boone et al. 2007; Hunt et al. 2008). The analysis of the torques exerted by the stellar gravitational potential on the molecular gas of the NUGA sample has shown that the gas can be driven away from the AGN (e.g. for NGC 4321) or toward it (e.g. for NGC 2782, NGC 3147, and NGC 4579). However, the velocities observed for NUGA are too small to correspond to the AGN feedback models where violent molecular outflows and superwinds are expected (e.g. Narayanan et al. 2006; Hopkins \& Hernquist 2006).

The different morphologies we find are probably related to the various timescales (García-Burillo et al. 2005). Large-scale bars can transport gas inward efficiently (e.g., Combes \& Gerin 1985; Sakamoto et al. 1999), and it appears that they can also drive powerful starbursts (e.g., Knapen et al. 2002; Jogee et al. 2005). Nevertheless, a clear correlation between large-scale bars and nuclear activity has not yet been found (e.g., Mulchaey \& Regan 1997), probably because the timescales for bar-induced gas inflow and AGN duty cycles are very different. Bars drive inflow over timescales ( $\gtrsim 300 \mathrm{Myr}$, Jogee et al. 2005) larger than those of AGN accretion-rate duty cycles ( 1-10 Myr, Heckman et al. 2004; Hopkins \& Hernquist 2006; King \& Pringle 2007), and active accretion seems to occur only intermittently over the lifetime of a galaxy (Ferrarese et al. 2001; Marecki et al. 2003; Janiuk et al. 2004; Hopkins \& Hernquist 2006; King \& Pringle 2007). This implies that most AGN are in an intermediate phase between active accretion episodes, making the detection of galaxies with nuclear accretion rather difficult.

Viscosity rather than self-gravity can also play a significant role in the fueling process. Viscous torques, generally weak and with timescales quite long at large radii, in combination with gravitational torques can coordinate efforts to produce recurrent episodes of activity during the typical lifetime of any galaxy (García-Burillo et al. 2005). Viscous torques can produce gas inflow on scales $\sim 100-200 \mathrm{pc}$ if they act on a contrasted nuclear ring distribution and in the absence of particularly strong positive gravitational torques. In NGC 4579, the efficiency of viscosity may be comparable to the efficiency of gravity torques in the inner $\sim 50$ pc (García-Burillo et al. 2009).

This paper, dedicated to the galaxy NGC 5953, is the latest of the NUGA series where results obtained for the galaxies of the sample are described on a case-by-case basis. NGC 5953 $\left(D=28 \mathrm{Mpc}\right.$ for $\left.H_{0}=73 \mathrm{~km} \mathrm{~s}^{-1} \mathrm{Mpc}^{-1}\right)$ is an interacting galaxy (e.g., Rampazzo et al. 1995; Casasola et al. 2004; Iono et al. 2005), classified as a Seyfert 2 by Gonzalez Delgado \& Perez (1996) and as a LINER by Veilleux et al. (1995), and of early and unbarred Hubble type (SAa pec). NGC 5953 and its late-type companion NGC 5954 (LINER/Seyfert 2, SAB(rs)cd pec) constitute a binary system (VV 244, Arp 91) where the two galaxies are separated by a projected distance of $5.8 \mathrm{kpc}\left(\sim 43^{\prime \prime}\right)$. They show clear signs of interaction visible in the distorted morphology, the presence of a tidal bridge (or distorted arm) connecting the two galaxies, and of prominent star-forming regions. Both galaxies have circumnuclear starbursts that may have been induced by the interaction (Gonzalez Delgado \& Perez 1996).

NGC 5953 hosts a compact radio core and jet, revealed by high-resolution radio continuum observations with MERLIN (Krips et al. 2007a). The jet is resolved at $18 \mathrm{~cm}$, and after beam deconvolution is roughly $\sim 0$ ' 3 in length $(40 \mathrm{pc})$, with a position
Table 1. Fundamental parameters for NGC 5953.

\begin{tabular}{lll}
\hline \hline Parameter & Value $^{b}$ & References $^{c}$ \\
\hline$\alpha_{\mathrm{J} 2000}{ }^{a}$ & $15^{\mathrm{h}} 34^{\mathrm{m}} 32.36^{\mathrm{s}}$ & $(1)$ \\
$\delta_{\mathrm{J} 2000}{ }^{a}$ & $15^{\circ} 11^{\prime} 37^{\prime \prime} .70$ & $(1)$ \\
$\alpha_{\text {dyn }}{ }^{a}$ & $15^{\mathrm{h}} 34^{\mathrm{m}} 32.38^{\mathrm{s}}$ & $(2)$ \\
$\delta_{\text {dyn }}{ }^{\circ}$ & $15^{\circ} 11^{\prime} 37^{\prime \prime} .59$ & $(2)$ \\
$V_{\text {sys,hel }}$ & $1990 \mathrm{~km} \mathrm{~s}{ }^{-1}$ & $(1)$ \\
RC3 Type & SAa pec & $(3)$ \\
Nuclear Activity & $\mathrm{S} 2 / \mathrm{LINER}$ & $(4) \&(5)$ \\
Inclination & $42^{\circ}$ & $(1)$ \\
Position Angle & $45^{\circ} \pm 1^{\circ}$ & $(1)$ \\
Distance & $28 \mathrm{Mpc}\left(1^{\prime \prime}=136 \mathrm{pc}\right)$ & $(3)$ \\
$L_{B}$ & $4.9 \times 10^{9} L_{\odot}$ & $(6)$ \\
$M_{\mathrm{HI}}$ & $1.1 \times 10^{9} M_{\odot}$ & $(7)$ \\
$M_{\mathrm{H}}$ & $2.2 \times 10^{9} M_{\odot}$ & $(8)$ \\
$M_{\text {dust }}(60$ and $100 \mu \mathrm{m})$ & $2.7 \times 10^{6} M_{\odot}$ & $(6)$ \\
$L_{\mathrm{FIR}}$ & $1.4 \times 10^{10} L_{\odot}$ & $(9)$ \\
\hline
\end{tabular}

Notes. ${ }^{(a)}\left(\alpha_{\mathrm{J} 2000}, \delta_{\mathrm{J} 2000}\right)$ is the phase tracking center of our ${ }^{12} \mathrm{CO}$ observations, $\left(\alpha_{\mathrm{dyn}}, \delta_{\mathrm{dyn}}\right)$ is the dynamical center derived from radio observations for the core of NGC 5953 by Krips et al. (2007a); ${ }^{(b)}$ luminosity and mass values extracted from the literature have been scaled to the distance of $D=28 \mathrm{Mpc}$; ${ }^{(c)}$ (1) this paper; (2) Krips et al. (2007a); (3) NASA/IPAC Extragalactic Database (NED); (4) Gonzalez Delgado \& Perez (1996); (5) Veilleux et al. (1995); (6) Casasola et al. (2004); (7) Haan et al. (2008); (8) Iono et al. (2005); (9) IRAS Catalog.

angle (PA) of $\sim 10^{\circ}$. The small-scale radio continuum structure at $20 \mathrm{~cm}$ is similar in orientation, but with lower spatial resolution $\left(\sim 1^{\prime \prime} .5\right)$, slightly more toward the east, PA $\sim 25^{\circ}$ (Jenkins 1984). This jet-like elongation as seen at lower resolution was first referred to as a "jet" by Gonzalez Delgado \& Perez (1996) because it is roughly aligned with the structure in the "excitation map", obtained by dividing [OIII] emission by $\mathrm{H} \alpha$.

The NGC 5953/54 pair has also been mapped in atomic and molecular gas. Both galaxies are embedded in a common HI envelope, with a clear velocity gradient along the HI plume extending more than $8 \mathrm{kpc}$ to the northwest (Chengalur et al. 1994; Iono et al. 2005; Haan et al. 2007, 2008). There is some indication of a faint diffuse optical counterpart to the HI plume (Chengalur et al. 1994; Hernández-Toledo et al. 2003). The Hi peak is also significantly displaced from the stellar disks (Iono et al. 2005). The overall HI velocity gradient runs from southeast to northwest, roughly perpendicular to the rotation in the ionized and molecular gas (Hernández-Toledo et al. 2003; Iono et al. 2005). The most recent HI mass determination for NGC 5953 has been obtained by Haan et al. (2008), $M_{\mathrm{HI}}=1.1 \times 10^{9} M_{\odot}$ (value scaled to our adopted distance of $D=28 \mathrm{Mpc}$ ), typical of that expected for interacting galaxies of the same morphological type (Casasola et al. 2004).

The molecular gas distribution in NGC 5953 is symmetric with a short extension pointing toward NGC 5954 (e.g., Yao et al. 2003; Iono et al. 2005). The $\mathrm{H}_{2}$ mass content estimated by Iono et al. (2005) is $2.2 \times 10^{9} M_{\odot}$ (scaled to the distance of $D=$ $28 \mathrm{Mpc}$ for NGC 5953), higher than the molecular hydrogen mass found for the interacting companion NGC $5954\left(M_{\mathrm{H}_{2}}=\right.$ $\left.1.1 \times 10^{9} M_{\odot}\right)$. Higher-order transitions of the $\mathrm{CO}$ molecule have also been detected in NGC 5953, including the ${ }^{12} \mathrm{CO}(3-2)$ line by Yao et al. (2003) with the James Clerk Maxwell Telescope $\left(F W H M \sim 15^{\prime \prime}\right)$, suggesting a high excitation of the carbon monoxide, indicative of dense and hot gas. Table 1 summarizes the fundamental characteristics of NGC 5953. 
The structure of this paper is as follows. In Sect. 2, we describe our new observations of NGC 5953 and the literature data with which we compare them. In Sects. 3 and 4, we present the observational results, both single dish and interferometric, describing morphology, excitation conditions, and kinematics of the molecular gas in the inner kpc of NGC 5953. Comparisons between ${ }^{12} \mathrm{CO}$ observations and those obtained at other wavelengths are given in Sect. 5. In Sect. 6, we describe the computation of the gravity torques derived from the stellar potential in the inner region of NGC 5953. Section 7 summarizes our results.

We will assume a distance to NGC 5953 of $D=28 \mathrm{Mpc}$ (HyperLeda ${ }^{1}$, Paturel et al. 2003) and a Hubble constant $H_{0}=$ $73 \mathrm{~km} \mathrm{~s}^{-1} \mathrm{Mpc}^{-1}$. This distance implies that $1^{\prime \prime}$ corresponds to $136 \mathrm{pc}$.

\section{Observations}

\subsection{Interferometric observations}

We observed NGC 5953 with the IRAM PdBI in the ABCD configuration of the array between September 2003 and February 2004 in the ${ }^{12} \mathrm{CO}(1-0)(115 \mathrm{GHz})$ and the ${ }^{12} \mathrm{CO}(2-1)(230 \mathrm{GHz})$ rotational transitions. The PdBI receiver characteristics, the observing procedures, and the image reconstruction are the same as described in García-Burillo et al. (2003). The quasars 3C454.3 and 3C273 were used for bandpass and flux calibrations respectively, and the quasar $1546+027$ was used for phase and amplitude calibrations.

Data cubes with $512 \times 512$ pixels $\left(0\right.$ ". 20 pixel $^{-1}$ for ${ }^{12} \mathrm{CO}(1-$ $0)$ and 0.14 pixel $^{-1}$ for ${ }^{12} \mathrm{CO}(2-1)$ ) were created over a velocity interval of $-242 \mathrm{~km} \mathrm{~s}^{-1}$ to $+242 \mathrm{~km} \mathrm{~s}^{-1}$ in bins of $5 \mathrm{~km} \mathrm{~s}^{-1}$. The images were reconstructed using the standard IRAM/GILDAS ${ }^{2}$ software (Guilloteau \& Lucas 2000) and restored with Gaussian beams of dimensions 2'. $0 \times 1$ 1'. $4(\mathrm{PA}=$ $\left.25^{\circ}\right)$ at $115 \mathrm{GHz}$ and $1^{\prime \prime} .1 \times 0.7\left(\mathrm{PA}=22^{\circ}\right)$ at $230 \mathrm{GHz}$. We used natural weighting to generate the ${ }^{12} \mathrm{CO}(1-0)$ maps and uniform weighting to generate ${ }^{12} \mathrm{CO}(2-1)$ maps. Such a procedure maximizes the flux recovered in ${ }^{12} \mathrm{CO}(1-0)$ and optimizes the spatial resolution in ${ }^{12} \mathrm{CO}(2-1)$. In the cleaned maps, the rms uncertainty $\sigma$ in $5 \mathrm{~km} \mathrm{~s}^{-1}$ width velocity channels is $2.7 \mathrm{mJybeam}^{-1}$ and $6.0 \mathrm{mJy}^{-1}$ beam $^{-1}$ for the ${ }^{12} \mathrm{CO}(1-0)$ and ${ }^{12} \mathrm{CO}(2-1)$ lines, respectively. At a level of $\sim 3 \sigma$ no $3 \mathrm{~mm}(1 \mathrm{~mm})$ continuum was detected toward NGC 5953, down to an rms noise level of $0.32 \mathrm{mJy}^{-1}$ beam $^{-1}\left(0.48 \mathrm{mJy}^{-}\right.$beam $\left.^{-1}\right)$. The conversion factors between intensity and brightness temperature are $32 \mathrm{~K}\left(\mathrm{Jy} \mathrm{beam}^{-1}\right)^{-1}$ at $115 \mathrm{GHz}$ and $28 \mathrm{~K}\left(\mathrm{Jy} \mathrm{beam}^{-1}\right)^{-1}$ at $230 \mathrm{GHz}$. All velocities are referred to the systemic velocity $V_{\text {sys,hel }}=1990 \mathrm{~km} \mathrm{~s}^{-1}$ (see later Sect. 4.1) and $(\Delta \alpha, \Delta \delta)$ offsets are relative to the phase tracking center of the observations $\left(\alpha_{J 2000}, \delta_{J 2000}\right)=\left(15^{\mathrm{h}} 34^{\mathrm{m}} 32.36^{\mathrm{s}}, 15^{\circ} 11^{\prime} 37^{\prime \prime} \cdot 70\right)$. All maps presented in this paper are centered on this position. The maps are not corrected for primary beam attenuation.

\subsection{Single dish observations and short spacing correction}

We performed IRAM $30 \mathrm{~m}$ telescope observations in a $5 \times 5$ raster pattern with $7^{\prime \prime}$ spacing from 16 to 19 July 2002. We used 4 SIS receivers to observe simultaneously at the frequencies of the ${ }^{12} \mathrm{CO}(1-0)(115 \mathrm{GHz})$, the ${ }^{12} \mathrm{CO}(2-1)(230 \mathrm{GHz})$, and the $\mathrm{HCN}(1-0)$ (89 GHz) lines. The half power beam widths are

\footnotetext{
${ }^{1}$ http://leda.univ-lyon $1 . f r$

${ }^{2}$ http://wWw.iram.fr/IRAMFR/GILDAS/
}

$22^{\prime \prime}$ for ${ }^{12} \mathrm{CO}(1-0), 12^{\prime \prime}$ for ${ }^{12} \mathrm{CO}(2-1)$, and $29^{\prime \prime}$ for $\mathrm{HCN}(1-$ $0)$. The $\mathrm{CO}(2-1)$ line has been observed in dual-polarization. Typical system temperatures were $\sim 270-390 \mathrm{~K}$ at $115 \mathrm{GHz}$, $\sim 300-750 \mathrm{~K}$ at $230 \mathrm{GHz}$, and $\sim 120 \mathrm{~K}$ at $89 \mathrm{GHz}$. Throughout this paper the line intensity scale is expressed in units of $T_{\mathrm{mb}}$, the beam-averaged radiation temperature. $T_{\mathrm{mb}}$ is related to $T_{A}^{*}$, the equivalent antenna temperature reported above the atmosphere, by $\eta=T_{\mathrm{A}}^{*} / T_{\mathrm{mb}}$ where $\eta$ is the telescope main-beam efficiency. At $115 \mathrm{GHz} \eta=0.79$, at $230 \mathrm{GHz} \eta=0.54$, and at $89 \mathrm{GHz} \eta=0.82$. All observations were performed in "wobblerswitching" mode, with a minimum phase time for spectral line observations of $2 \mathrm{~s}$ and a maximum beam throw of $240^{\prime \prime}$. The pointing accuracy was $\sim 3^{\prime \prime}$ rms. The single dish maps are centered on the phase tracking center of the interferometric observations (see Table 1).

Single dish ${ }^{12} \mathrm{CO}$ observations were used to compute short spacings and complete the interferometric measurements. We combined $30 \mathrm{~m}$ and PdBI data using the SHORT-SPACE task available in the GILDAS software. The best compromise between good angular resolution and complete restoration of the missing extended flux was found by varying the relative weights of $30 \mathrm{~m}$ and PdBI observations. These were chosen in order to obtain the same mean weights in the single dish data as in the interferometric data within a ring in the $u v$ plane ranging from $1.25 D / \lambda$ to $2.5 D / \lambda(D=15 \mathrm{~m})$. The combined PdBI+30 $\mathrm{m}$ data have produced maps with angular resolutions of $2^{\prime \prime} 1 \times 1$ ". 4 at $\mathrm{PA}=25^{\circ}$ for the ${ }^{12} \mathrm{CO}(1-0)$ and $1^{\prime \prime} .1 \times 0.7$ at $\mathrm{PA}=22^{\circ}$ for the ${ }^{12} \mathrm{CO}(2-1)$. In the combined maps, the rms uncertainty $\sigma$ in $5 \mathrm{~km} \mathrm{~s}^{-1}$ width velocity channels is $2.5 \mathrm{mJy}^{-1} \mathrm{beam}^{-1}$ and $5.5 \mathrm{mJy}$ beam $^{-1}$ for the ${ }^{12} \mathrm{CO}(1-0)$ and ${ }^{12} \mathrm{CO}(2-1)$ lines, respectively. All figures presented in this paper are made with shortspacing-corrected data.

We estimate that the ${ }^{12} \mathrm{CO}(1-0)$ map including only PdBI observations within $\sim 22^{\prime \prime}\left({ }^{12} \mathrm{CO}(1-0) \mathrm{HPBW}\right.$ for the $30 \mathrm{~m}$ telescope) recovers a flux of $S_{\mathrm{CO}(1-0)}=150 \mathrm{Jy} \mathrm{km} \mathrm{s}^{-1}, \sim 81 \%$ of the total flux measured with the combined PdBI $+30 \mathrm{~m}$ map, $S_{\mathrm{CO}(1-0)}=185 \mathrm{Jy} \mathrm{km} \mathrm{s}^{-1}$. Table 2 collects ${ }^{12} \mathrm{CO}(1-0)$ flux values, both present in literature and determined with our observations, for NGC 5953. In this table, Col. (1) indicates the reference, Cols. (2) and (3) the telescope, both single dish and interferometer, and the diameter of the single dish telescope, Col. (4) is the primary beam of the instrument or the diameter used for the performed photometry, Col. (5) is the beam in interferometric measurements, and Col. (6) is the measured flux. Table 2 shows that ${ }^{12} \mathrm{CO}(1-0)$ fluxes we obtained with interferometric observations, single dish, and combined measurements $(\mathrm{PdBI}+30 \mathrm{~m})$ are in good mutual agreement with each other and with data present in literature. Our ${ }^{12} \mathrm{CO}(1-0)$ combined map within $42^{\prime \prime}$ gives a value $\left(254 \mathrm{Jy} \mathrm{km} \mathrm{s}^{-1}\right)$ consistent with the flux of $233 \mathrm{Jy} \mathrm{km} \mathrm{s}^{-1}$ found with OVRO (Iono et al. 2005), but consistent also with the NRAO flux of $365 \mathrm{Jy} \mathrm{km} \mathrm{s}^{-1}$ (Zhu et al. 1999) taking into account that they used a single dish with a diameter of $12 \mathrm{~m}$ and a primary beam of 55". In addition, we recovered $\sim 79 \%$ of the total flux detected by Young et al. (1995) with the FCRAO (320 Jy km s${ }^{-1}$ ), a reasonable agreement considering the uncertainties in the amplitude calibration and the non-correction by the primary beam attenuation.

Figures 1 and 2 show the channel maps for the ${ }^{12} \mathrm{CO}(1-$ $0)$ and ${ }^{12} \mathrm{CO}(2-1)$ lines, respectively, in the central region of NGC 5953. Figures 4 and 5 display the single dish data, for the ${ }^{12} \mathrm{CO}(1-0),{ }^{12} \mathrm{CO}(2-1)$, and $\mathrm{HCN}(1-0)$ lines. The two ${ }^{12} \mathrm{CO}$ lines have been mapped on a $5 \times 5$ grid with $7^{\prime \prime}$ spacings, while the $\mathrm{HCN}(1-0)$ line has been mapped on a $3 \times 3$ grid with 7 " spacings 
Table 2. ${ }^{12} \mathrm{CO}(1-0)$ flux values for NGC 5953.

\begin{tabular}{llllll}
\hline \hline Reference & Telescope & $\begin{array}{l}\text { Diameter } \\
{[\mathrm{m}]}\end{array}$ & $\begin{array}{l}\text { Primary beam or FOV } \\
{\left[{ }^{a}\right]}\end{array}$ & $\begin{array}{l}\text { Beam } \\
{\left[{ }^{\prime \prime} \times{ }^{\prime \prime}\right]}\end{array}$ & $\begin{array}{l}\text { Flux } \\
{\left[\mathrm{Jy} \mathrm{km} \mathrm{s}^{-1}\right]}\end{array}$ \\
\hline Iono et al. (2005) & OVRO & & 60 & $4.4 \times 3.6$ & 233 \\
Zhu et al. (1999) & NRAO & 12 & 55 & & 365 \\
Young et al. (1995) & FCRAO & 14 & 45 & $2.1 \times 1.4$ & 254 \\
This paper & PdBI+30 m & & 42 & $2.1 \times 1.4$ & 185 \\
This paper & PdBI+30 m & & $22^{\mathrm{b}}$ & $2.0 \times 1.4$ & 150 \\
This paper & PdBI & & $22^{\mathrm{b}}$ & & $167^{\mathrm{c}}$ \\
This paper & $30 \mathrm{~m}$ & 30 & $22($ central position) & & $377^{\mathrm{d}}$ \\
This paper & $30 \mathrm{~m}$ & 30 & $22\left(\right.$ inner $\left.50^{\prime \prime} \times 50^{\prime \prime}\right)$ & & \\
\hline
\end{tabular}

Notes. ${ }^{(a)}$ Primary beam is considered for single dish observations, while field-of-view (FOV) for interferometric or combined (interferometric+single dish) ones; ${ }^{(b)}$ the photometry has been performed within $22^{\prime \prime}$, the ${ }^{12} \mathrm{CO}(1-0)$ primary beam for the $30 \mathrm{~m}$ telescope; ${ }^{(c)}$ the ${ }^{12} \mathrm{CO}(1-0)$ recovered flux for the central position $\left(0^{\prime \prime}, 0^{\prime \prime}\right) ;{ }^{(d)}$ the ${ }^{12} \mathrm{CO}(1-0)$ recovered flux for inner $50^{\prime \prime} \times 50^{\prime \prime}, 5 \times 5$ mapping with $7^{\prime \prime}$ spacing (see Sect. 2.2$)$.

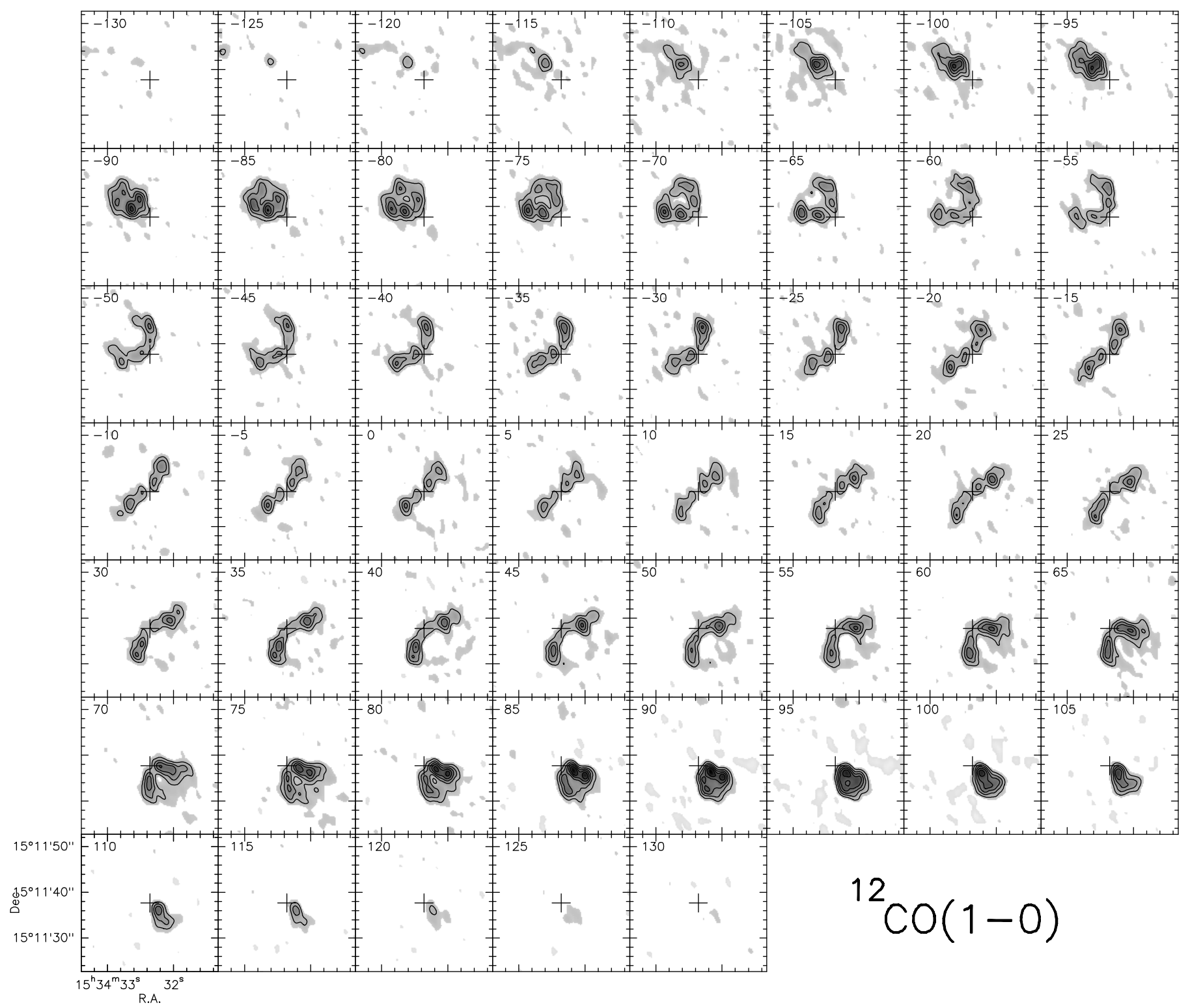

Fig. 1. ${ }^{12} \mathrm{CO}(1-0)$ velocity channel maps observed with the IRAM PdBI+30 $\mathrm{m}$ in the nucleus of NGC 5953, with a spatial resolution of 2 .' $1 \times$ 1.' $4(H P B W)$. The maps are centered on the phase tracking center of our observations assumed to be coincident with the dynamical center of the galaxy (see Sect. 4.1). Velocity channels range from $\Delta V=-130 \mathrm{~km} \mathrm{~s}^{-1}$ to $+130 \mathrm{~km} \mathrm{~s}^{-1}$ in steps of $5 \mathrm{~km} \mathrm{~s}^{-1}$ relative to $V_{\text {sys,hel }}=1990 \mathrm{~km} \mathrm{~s}{ }^{-1}$ (see Sect. 4.1). The contours run from $-0.20 \mathrm{mJy}_{\text {beam }}{ }^{-1}$ to $120 \mathrm{mJy}$ beam ${ }^{-1}$ with spacings of $20 \mathrm{mJy}$ beam $^{-1}$. 


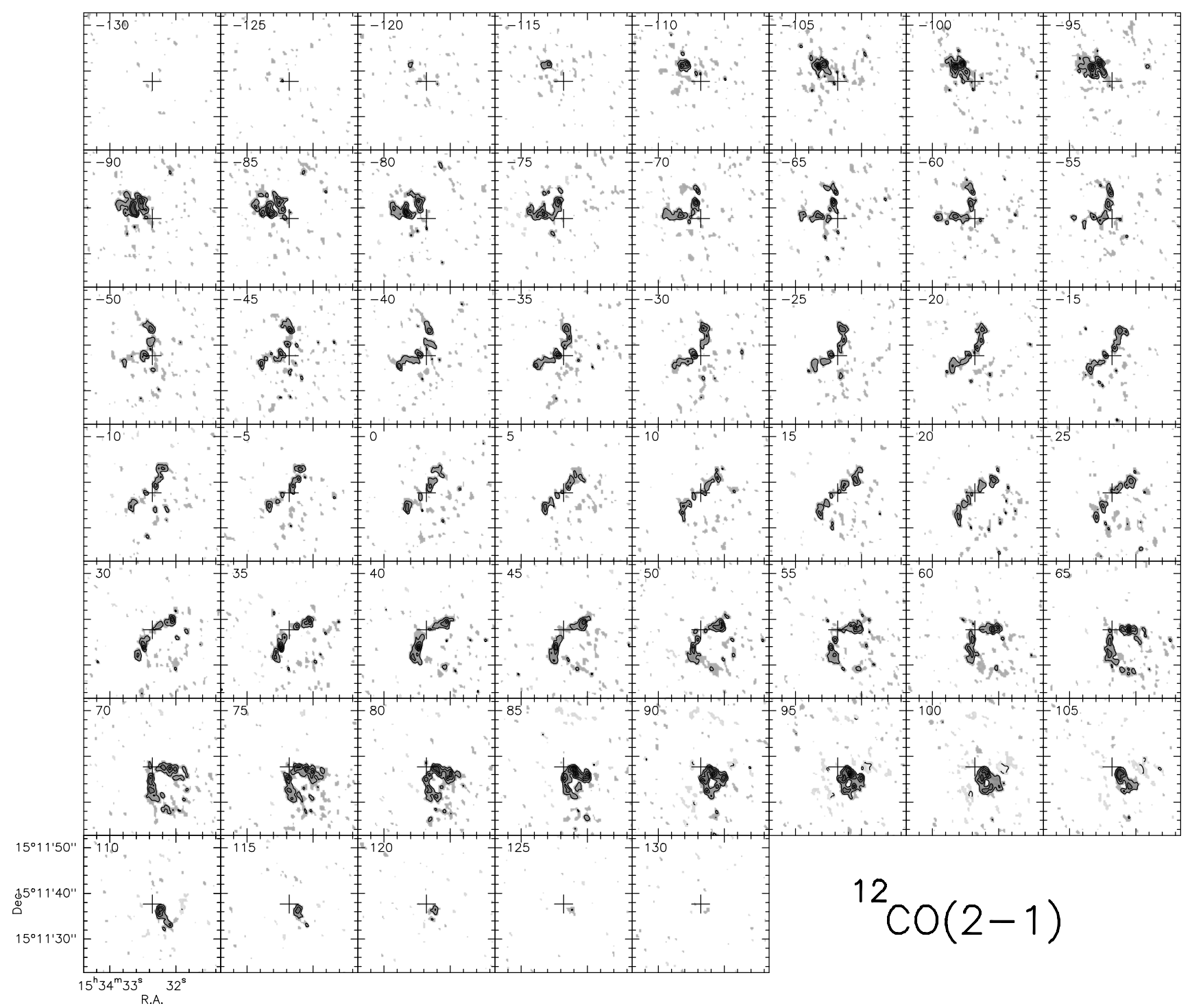

Fig. 2. Same as Fig. 1 but for the ${ }^{12} \mathrm{CO}(2-1)$ line, with a spatial resolution of $11^{\prime \prime} 1 \times 0$.' 7 . The contours run from $-60 \mathrm{mJy}$ beam $^{-1}$ to $150 \mathrm{mJy}$ beam ${ }^{-1}$ with spacings of $30 \mathrm{mJy}^{\text {beam }}{ }^{-1}$.

and the nine $\mathrm{HCN}(1-0)$ spectra have been averaged to improve the signal-to-noise.

\subsection{Optical and infrared images}

We first acquired a broad-band optical image from the HST archive $^{3}$ of NGC 5953 obtained with the $F 606 \mathrm{~W}$ filter (mean wavelength of $5940 \AA$ ). This image was first published by Malkan et al. (1998), in a survey of 256 of the nearest $(z \leq 0.035)$ Seyfert 1, Seyfert 2, and starburst galaxies. The image covers the inner $\sim 20^{\prime \prime} \times 20^{\prime \prime}$ with a pixel size of $0{ }^{\prime} 045$.

From the HST archive, we also acquired the HST NICMOS F160W ( $H$-band, $1.6 \mu \mathrm{m})$ image of NGC 5953. This nearinfrared (NIR) image was presented by Regan \& Mulchaey (1999) and Hunt \& Malkan (2004), and covers the inner $\sim 19^{\prime \prime} \times$ $19^{\prime \prime}$ of the galaxy with a pixel size of $0{ }^{\prime} 075$.

\footnotetext{
3 Based on observations made with the NASA/ESA Hubble Space Telescope, and obtained from the Hubble Legacy Archive, which is a collaboration between the Space Telescope Science Institute (STScI/NASA), the Space Telescope European Coordinating Facility (ST-ECF/ESA) and the Canadian Astronomy Data Centre (CADC/NRC/CSA).
}

We also acquired infrared (IR) images obtained with the IRAC camera on Spitzer, available thanks to the project "Starburst Activity in Nearby Galaxies" (Principal Investigator: G. Rieke). The IRAC images, from 3.6, to $8 \mu \mathrm{m}$, were reduced with MOPEX (Makovoz \& Marleau 2005) which accounts for distortion and rotates to a fiducial coordinate system. They cover a large sky area $\left(\sim 320^{\prime \prime} \times 320^{\prime \prime}\right)$ including both NGC 5953 and NGC 5954. We imposed a pixel size of 1".20 for the final images, roughly the same as the original IRAC detector. Following Helou et al. (2004), a "dust-only" (non-stellar) image was derived from the $8 \mu \mathrm{m}$ image, with subtraction of the stellar component computed by scaling the $3.6 \mu \mathrm{m}$ and $4.5 \mu \mathrm{m}$ images and subtracting them. This image will be referred to as a dust-only image, and should be dominated by emission from Polycyclic Aromatic Hydrocarbons (PAHs) and perhaps some hot dust continuum emission.

A $J-K$ image was derived from NIR data published by Hunt et al. (1999) acquired with ARNICA (Arcetri Near-Infrared Camera) mounted on the Nordic Optical Telescope $\left(\mathrm{NOT}^{4}\right)$.

4 The NOT is operated on the island of La Palma jointly by Denmark, Finland, Norway, and Sweden, in the Spanish Observatorio del Roque de los Muchachos of the Instituto de Astrofisica de Canarias. 

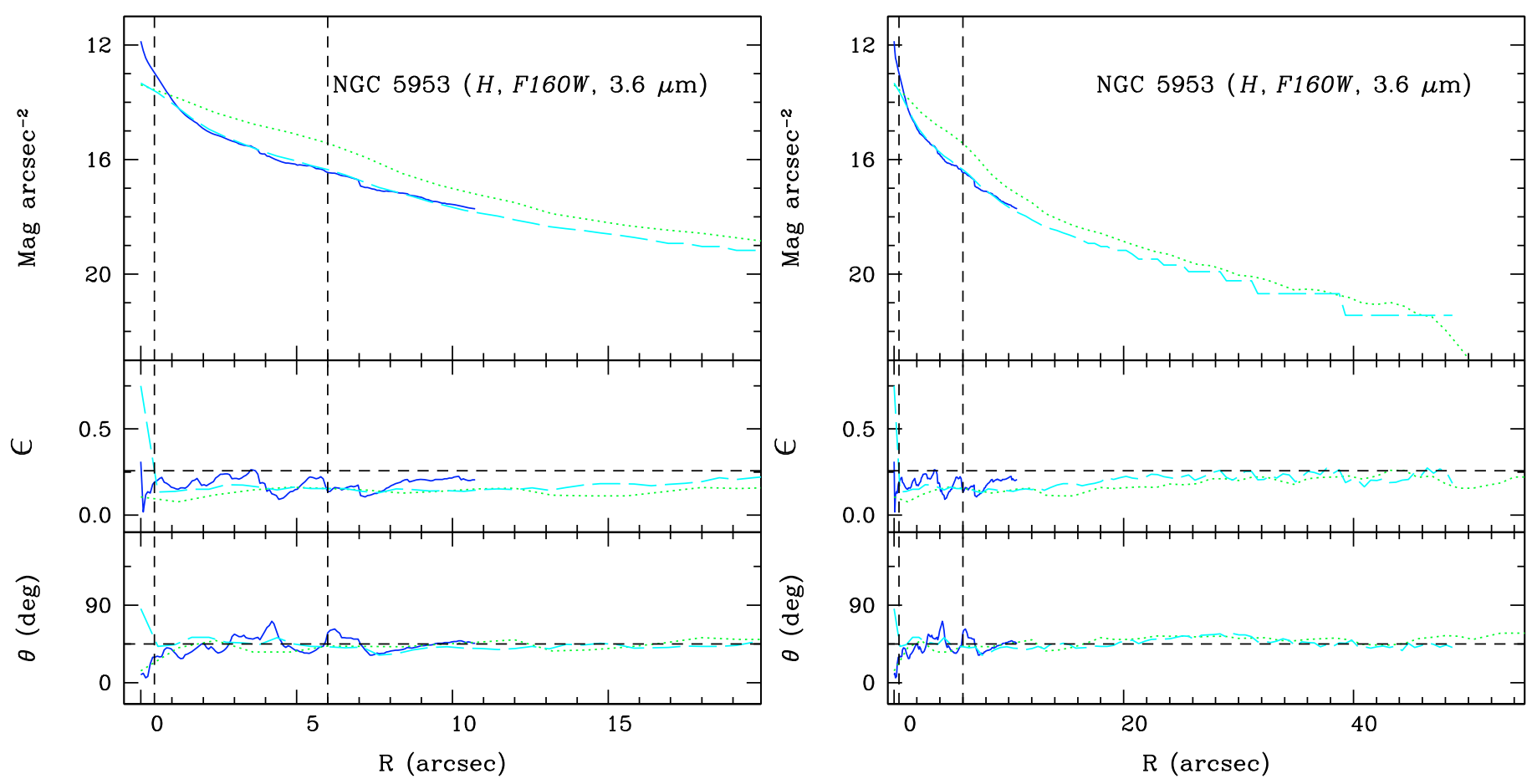

Fig. 3. Radial surface brightness profiles of NGC 5953 made by elliptical averages. The dotted line corresponds to IRAC $3.6 \mu \mathrm{m}$, the dashed line to ground-based $H$-band, and the solid line to HST/NICMOS/F160W. The left panel shows a smaller FOV than the right panel. The adopted position angle $\left(45^{\circ}\right)$ and the best-fit inclination $\left(42^{\circ}\right)$ are shown by dashed horizontal lines in the lower panels (see Sect. 4.1). The dashed vertical lines correspond to the $60 \mathrm{pc}\left(\sim 00^{\prime} 44\right.$, radius) nuclear bar seen in the HST F606W image (see Sect. 5.1), and the 820 pc (radius) ring observed in the residuals of the bulge-disk decomposition (see Sect. 5.2).

ARNICA was an imaging camera for the NIR bands between 1.0 and $2.5 \mu \mathrm{m}$ based on the Rockewell HgTeCd-array detector NICMOS $3(256 \times 256$ pixels $)$, and designed and built by Arcetri Observatory (Firenze, Italy) for the Infrared Telescope at Gornergrat in Switzerland. The ARNICA/NOT NIR images $(J, H, K)$ cover $\sim 120^{\prime \prime} \times 120^{\prime \prime}$, including the pair of interacting galaxies, NGC 5953 and NGC 5954, with a pixel size of 0.'546.

We compared the surface brightness profiles of the groundand space-based images by extracting elliptically averaged profiles, centered on the brightness peaks. The position angle and ellipticity were allowed to vary in the ellipse fitting. These radial profiles are shown in Fig. 3; the dashed horizontal lines in the lower panels correspond to the adopted position angle and inclination (see Sect. 4.1). The profiles agree quite well; in particular the ground-based $H$-band and HST F160W show the same trend down to the nuclear regions where the ground-based atmospheric beam smearing leads to lower surface brightness. The $H$-band/3.6 $\mu \mathrm{m}$ color is relatively constant throughout the entire radial range shown, with slightly redder colors from $\sim 5-8^{\prime \prime}$.

\section{Single dish results}

The observations performed with the $\mathrm{A}$ and $\mathrm{B}$ receivers of the IRAM $30 \mathrm{~m}$ telescope in the two ${ }^{12} \mathrm{CO}$ lines covered the inner $\sim 50^{\prime \prime}$, corresponding to the central $\sim 6.8 \mathrm{kpc}$ (in diameter) of the galaxy (Fig. 4). The 25 observed positions show that the central region of NGC 5953 hosts extended molecular emission both in ${ }^{12} \mathrm{CO}(1-0)$ and ${ }^{12} \mathrm{CO}(2-1)$ emission (Fig. 4). The maximum detected $T_{\mathrm{mb}}$ is $0.18 \mathrm{~K}$ in ${ }^{12} \mathrm{CO}(1-0)$ in the southwest corresponding to offset position $\left(-7^{\prime \prime},-7^{\prime \prime}\right)$, and $0.54 \mathrm{~K}$ in ${ }^{12} \mathrm{CO}(2-1)$ at the same offset position $\left(-7^{\prime \prime},-7^{\prime \prime}\right)$.
Within the inner $\sim 50^{\prime \prime} \times 50^{\prime \prime}$ we estimate a flux of $377 \mathrm{Jy} \mathrm{km} \mathrm{s}^{-1}$, in good agreement with previous single dish flux determinations (Zhu et al. 1999; Young et al. 1995, see Table 2). From this ${ }^{12} \mathrm{CO}(1-0)$ integrated flux assuming a $\mathrm{H}_{2}-\mathrm{CO}$ conversion factor $X=N\left(\mathrm{H}_{2}\right) / I_{\mathrm{CO}}=2.2 \times 10^{20} \mathrm{~cm}^{-2}\left(\mathrm{~K} \mathrm{~km} \mathrm{~s}^{-1}\right)^{-1}$ (Solomon \& Barrett 1991), we can derive the $\mathrm{H}_{2}$ mass within the observed region as:

$$
M_{\mathrm{H}_{2}}\left[M_{\odot}\right]=8.653 \times 10^{3} D^{2}[\mathrm{Mpc}] S_{\mathrm{CO}(1-0)}\left[\mathrm{Jy} \mathrm{km} \mathrm{s}^{-1}\right] .
$$

We obtain $M_{\mathrm{H}_{2}} \sim 2.6 \times 10^{9} M_{\odot}$, and including the mass of helium, the corresponding total molecular mass is $M_{\mathrm{mol}}=$ $M_{\mathrm{H}_{2}+\mathrm{He}}=1.36 \times M_{\mathrm{H}_{2}} \sim 3.5 \times 10^{9} M_{\odot}$.

The $\mathrm{HCN}(1-0)$ line has been observed for 9 positions with $7 "$ spacing, covering the central $\sim 43^{\prime \prime}(\sim 5.8 \mathrm{kpc})$. The $\mathrm{HCN}(1-0)$ emission is detected in the west part of the observed region, and the average spectrum over the $3 \times 3$ grid is shown in Fig. 5. The $\mathrm{HCN}(1-0)$ peak is at $T_{\mathrm{mb}} \sim 0.005 \mathrm{~K}$. The $\mathrm{CO}(1-$ $0) / \mathrm{HCN}(1-0)$ ratio is very high, equal to 20 on average over the center, where instead we would expect an enhanced HCN emission due to the AGN and thus a lower ratio. A high $\mathrm{CO} / \mathrm{HCN}$ line ratio is rarely encountered in AGN but not completely unusual. NGC 6951 has a ratio of 30 in the starburst ring and 2.5 in the nucleus (Krips et al. 2007b), and NGC 3147 of 20 in the inner $4 \mathrm{kpc}$ where the $\mathrm{CO}$ emission exhibits two ring-like structures around the nucleus (Casasola et al. 2008). On the other hand, the galaxy NGC 1097 has a CO/HCN ratio which ranges from 3 in the nucleus to 10 in the star-forming ring (Kohno et al. 2003). Since AGN activity implies an enhanced $\mathrm{HCN}$ emission relative to $\mathrm{CO}$ emission associated with the star-formation process, the high $\mathrm{CO}(1-0) / \mathrm{HCN}(1-0)$ ratio observed in NGC 5953 implies that excitation by star-formation is dominant over AGN excitation in the circumnuclear region. 

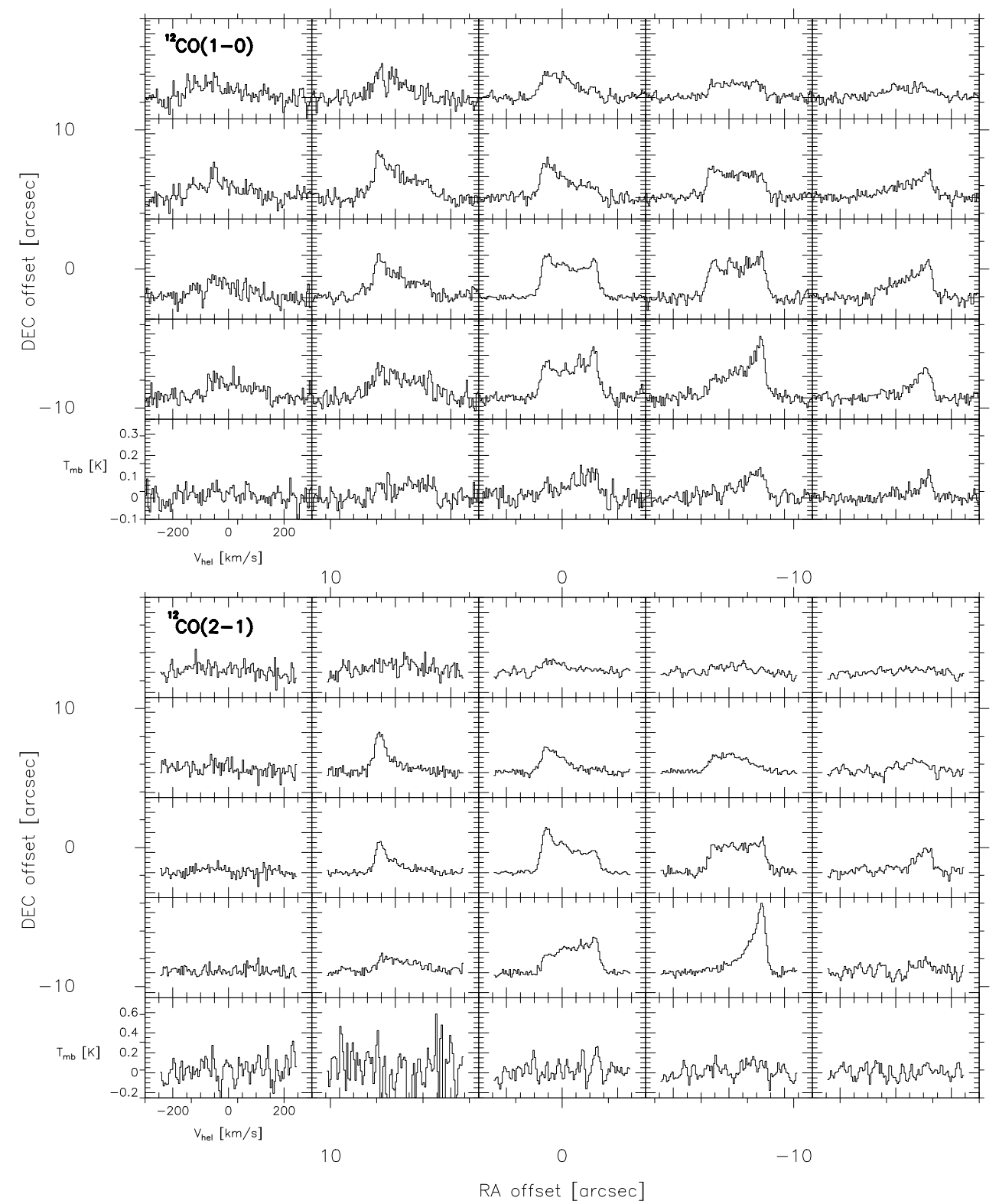

Fig. 4. Spectra maps of NGC 5953 made with the IRAM $30 \mathrm{~m}$ with 7 " spacing in ${ }^{12} \mathrm{CO}(1-0)($ top $)$ and ${ }^{12} \mathrm{CO}(2-1)$ (bottom). The positions are arcsec offsets relative to the phase tracking center of our observations. Each spectrum has a velocity scale from -300 to $300 \mathrm{~km} \mathrm{~s}^{-1}$, and a beam-averaged radiation temperature scale $\left(T_{\mathrm{mb}}\right)$ from -0.10 to $0.37 \mathrm{~K}$ for ${ }^{12} \mathrm{CO}(1-0)$ and from -0.25 to $0.75 \mathrm{~K}$ for ${ }^{12} \mathrm{CO}(2-1)$.

\section{Interferometric results}

\subsection{Dynamical center and inclination}

We assume that the dynamical center of NGC 5953 coincides with the position of the AGN "core" derived from radio observations (MERLIN/18 cm) by Krips et al. (2007a): $\alpha=$ $15^{\mathrm{h}} 34^{\mathrm{m}} 32.38^{\mathrm{s}}$ and $\delta=15^{\circ} 11^{\prime} 37^{\prime \prime} .59$. Since these coordinates are nearly coincident with those of the phase tracking center of our ${ }^{12} \mathrm{CO}$ observations (see Table 1), in the following we assume that our observations are centered on the dynamical center of the galaxy.

The spectral correlators were centered at $114.520 \mathrm{GHz}$ and $229.037 \mathrm{GHz}$ for the ${ }^{12} \mathrm{CO}(1-0)$ and ${ }^{12} \mathrm{CO}(2-1)$ line respectively, corresponding to $V_{\mathrm{LSR}}=1966 \mathrm{~km} \mathrm{~s}^{-1}$. The difference between LSR and heliocentric velocity is $16 \mathrm{~km} \mathrm{~s}^{-1}$, and therefore the observations were centered on $V_{\text {hel }}(\mathrm{PdBI})=1950 \mathrm{~km} \mathrm{~s}^{-1}$. In the inner $\sim 4^{\prime \prime}$ the velocity centroid is $50 \mathrm{~km} \mathrm{~s}^{-1}$ redshifted with respect to the heliocentric velocity of the center of our ${ }^{12} \mathrm{CO}(1-$ 0) observations (Fig. 6, left panel, one component Gaussian fit), and $30 \mathrm{~km} \mathrm{~s}^{-1}$ redshifted relative to ${ }^{12} \mathrm{CO}(2-1)$ (Fig. 6, right panel, one component Gaussian fit). We therefore estimate the systemic heliocentric velocity as the intermediate value between that determined for the ${ }^{12} \mathrm{CO}(1-0)$ and that for the ${ }^{12} \mathrm{CO}(2-1)$, $V_{\text {sys,hel }}=1990 \mathrm{~km} \mathrm{~s}^{-1}$.

In Figs. 6, we fitted the emissions also considering three Gaussian components, visible both in ${ }^{12} \mathrm{CO}(1-0)$ and in ${ }^{12} \mathrm{CO}(2-$ 1). These (red) fits show that the wing components for the two ${ }^{12} \mathrm{CO}$ lines are approximately at the same velocities within the noise $\left(-52 \mathrm{~km} \mathrm{~s}^{-1}\right.$ for the ${ }^{12} \mathrm{CO}(1-0)$ vs. $-57 \mathrm{~km} \mathrm{~s}^{-1}$ for the ${ }^{12} \mathrm{CO}(2-1)$, and $118 \mathrm{~km} \mathrm{~s}^{-1}$ for the ${ }^{12} \mathrm{CO}(1-0)$ vs. $113 \mathrm{~km} \mathrm{~s}^{-1}$ for the $\left.{ }^{12} \mathrm{CO}(2-1)\right)$. The major difference is present for the central Gaussian component, $33 \mathrm{~km} \mathrm{~s}^{-1}$ for the ${ }^{12} \mathrm{CO}(1-0)$ vs. $18 \mathrm{~km} \mathrm{~s}^{-1}$ for the ${ }^{12} \mathrm{CO}(2-1)$, maybe due to the clumpy nature of the inner molecular gas. The line ratio $R_{21}\left(I_{\mathrm{CO}(2-1)} / I_{\mathrm{CO}(1-0)}\right)$ assumes the 


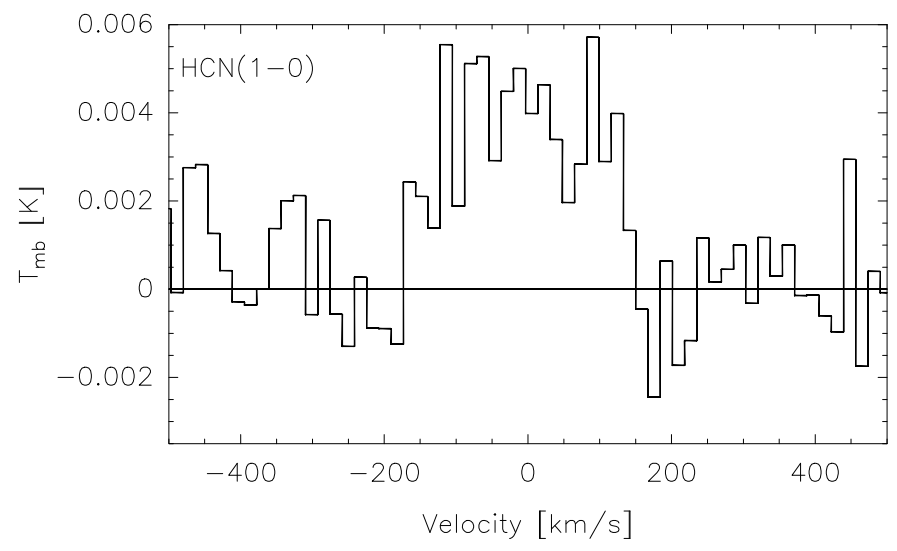

Fig. 5. HCN(1-0) spectrum toward the center of NGC 5953, averaged over the 9-point map made with the IRAM $30 \mathrm{~m}$ with $7^{\prime \prime}$ spacing. The spectrum has a velocity scale from -500 to $500 \mathrm{~km} \mathrm{~s}^{-1}$ and a beamaveraged radiation temperature scale $\left(T_{\mathrm{mb}}\right)$ from -0.0035 to $0.006 \mathrm{~K}$.

values of $1.2,0.6$, and 0.5 respectively for the three components passing from negative to positive velocities. The mean ratio of 0.8 is consistent with the line ratio discussed later in Sect. 4.3.

The systemic heliocentric velocity of $1990 \mathrm{~km} \mathrm{~s}^{-1}$ is $25 \mathrm{~km} \mathrm{~s}^{-1}$ redshifted with respect to the value determined from HI observations (1965 $\mathrm{km} \mathrm{s}^{-1}$, HyperLeda), and in agreement with the shift of $50 \mathrm{~km} \mathrm{~s}^{-1}$ inferred from previous ${ }^{12} \mathrm{CO}(1-0)$ observations with OVRO (Iono et al. 2005). This discrepancy between systemic velocity derived from ${ }^{12} \mathrm{CO}$ and $\mathrm{HI}$ observations is not unusual, especially in galaxies with a lopsided HI morphology. The NUGA galaxy NGC 4579 exhibits a difference of $\sim 50 \mathrm{~km} \mathrm{~s}^{-1}$, probably due to the interaction history of the galaxy and the different effect of the ram-pressure on the atomic and molecular gas (García-Burillo et al. 2009). Since the atomic gas is much more affected by ram-pressure (Kenney \& Young 1986; Vollmer et al. 2001) than the molecular component, the HI kinematics are more sensitive than the $\mathrm{CO}$ to the interaction history; NGC 5953 could present a similar scenario to NGC 4579. In fact, in NGC 5953 the HI has been detected mainly in the outer parts where a big tidal tail connecting the two galaxies shifts the $\mathrm{HI}$ barycenter with respect to the molecular gas that traces the less perturbed, inner parts of the galaxy.

We also derived the inclination of NGC 5953 by fitting the two-dimensional bulge/disk (B/D) decomposition on the ground-based $H$-band image and the IRAC $3.6 \mu \mathrm{m}$ image (see Sect. 5.2). The best-fit inclinations are $i=39^{\circ}$ and $46^{\circ}$, respectively; we therefore used a roughly intermediate value of $42^{\circ}$, which approximates quite well the outer regions of the NIR images (see Fig. 3).

\subsection{CO morphology and mass}

The ${ }^{12} \mathrm{CO}(1-0)$ and ${ }^{12} \mathrm{CO}(2-1)$ integrated intensity distributions are shown in Fig. 7. The $\mathrm{CO}$ emission is distributed over a disk of $\sim 16^{\prime \prime}(\sim 2.2 \mathrm{kpc})$ diameter. Our ${ }^{12} \mathrm{CO}(1-0)$ observations show several peaks, distributed more or less randomly, with the strongest one offset from the nucleus $\sim 2^{\prime \prime}$ toward the west/southwest, away from NGC 5954. The ${ }^{12} \mathrm{CO}(1-$ 0 ) distribution is different from the HI morphology, where the strongest emission has been detected mostly on the side nearer to NGC 5954 (e.g., Iono et al. 2005), although the HI resolution is probably insufficient to resolve distinct peaks. In the ${ }^{12} \mathrm{CO}(2-$ 1) map the central emission is also clearly resolved and more clumpy than in ${ }^{12} \mathrm{CO}(1-0)$. The strongest ${ }^{12} \mathrm{CO}(2-1)$ peak is not that at $\sim 2-3$ " in the west/southwest direction from the nucleus, like for ${ }^{12} \mathrm{CO}(1-0)$, but that at $\sim 1^{\prime \prime} .5$ in the east direction from the nucleus.

The total $\mathrm{H}_{2}$ mass derived from our interferometric $(\mathrm{PdBI}+30 \mathrm{~m})$ map within the $42^{\prime \prime}$ primary beam field of the PdBI $\left(S_{\mathrm{CO}}=254 \mathrm{Jy} \mathrm{km} \mathrm{s}{ }^{-1}\right.$, see Table 2$)$ is $M_{\mathrm{H}_{2}} \sim 1.7 \times 10^{9} M_{\odot}$ $\left(M_{\mathrm{mol}} \sim 2.3 \times 10^{9} M_{\odot}\right)$. Within a diameter of $22^{\prime \prime}\left(S_{\mathrm{CO}}=185 \mathrm{Jy}\right.$ $\mathrm{km} \mathrm{s}^{-1}$, see Table 2), we derive a $\mathrm{H}_{2}$ mass of $M_{\mathrm{H}_{2}} \sim 1.3 \times 10^{9} M_{\odot}$, $\sim 50 \%$ of the single dish $30 \mathrm{~m} \mathrm{H}_{2}$ mass (see Sect. 3) corresponding to a region about 6.6 times larger in area. Hence, half of the molecular gas is concentrated in the central region of NGC 5953, as expected for spiral galaxies. The molecular gas distribution consists mainly of individual giant molecular cloud complexes, with the biggest one alone having a mass of $M_{\mathrm{H}_{2}}=3.0 \times 10^{8} M_{\odot}$, while the smaller ones have masses of a few $10^{7} M_{\odot}$.

NGC 5953 is quite massive in molecular gas compared to the other NUGA galaxies, where typically the mass is on the order of $\sim 3 \times 10^{8} M_{\odot}$. Three galaxies of the NUGA sample, NGC 4569 (Boone et al. 2007), NGC 2782 (Hunt et al. 2008), NGC 3147 (Casasola et al. 2008), with molecular masses in the range of 1$3 \times 10^{9} M_{\odot}$, are comparable with NGC 5953. The extraordinary case of NGC 1961 (Combes et al. 2009) is excluded from these considerations: NGC 1961 exhibits a $\mathrm{H}_{2}$ mass of $\sim 1.8 \times 10^{10} M_{\odot}$, almost an order of magnitude more massive than any NUGA galaxy.

\subsection{CO line ratio}

The comparison between the two ${ }^{12} \mathrm{CO}$ maps, obtained after convolving the ${ }^{12} \mathrm{CO}(2-1)$ map to the lower resolution of the ${ }^{12} \mathrm{CO}(1-0)$ one and including short spacings for both maps, gives information about the local excitation conditions of the molecular gas. Figure 8 shows the ratio of the ${ }^{12} \mathrm{CO}(1-0)$ to ${ }^{12} \mathrm{CO}(2-1)$ convolved to the same resolution with ${ }^{12} \mathrm{CO}(1-0)$ contours as in Fig. 7 (left panel). The line ratio ranges from 0.3 to 0.9 inside the observed region and the bulk of the emission has a ratio between 0.4 and 0.8 , values consistent with optically thick emission in spiral disks (e.g., Braine \& Combes 1992; García-Burillo et al. 1993). Ratio values of $\sim 0.9$ are reached in some clumpy structures, randomly distributed inside the mapped region, especially those toward $\mathrm{E} / \mathrm{NE}$ with respect to the nucleus, well visible in ${ }^{12} \mathrm{CO}(2-1)$ emission (Fig. 7, right panel). A $R_{21}$ line ratio of 0.9 suggests a moderately high ${ }^{12} \mathrm{CO}$ excitation consistently with Yao et al. (2003) who have detected the ${ }^{12} \mathrm{CO}(3-2)$ line $(345.80 \mathrm{GHz})$ with an intensity of $I_{32}=38.7 \mathrm{~K} \mathrm{~km} \mathrm{~s}^{-1}$ and a line ratio $R_{32}=I_{32} / I_{10}=0.85$ in the nucleus of NGC 5953 . Different excitation conditions of the molecular gas appear to characterize the interacting companion galaxy NGC 5954, where the ${ }^{12} \mathrm{CO}(3-2)$ line has been detected with a ratio of $R_{32}=0.37$ (Yao et al. 2003).

\subsection{Kinematics}

The velocity-channel maps (see Figs. 1 and 2) show a general regularity of the large scale kinematics, typical for a rotating disk. Some local wiggles, not forming a coherent grand design, are superimposed on this regular pattern, both at negative and positive velocities especially to the west of the nucleus. These kinematic "glitches" are probably associated with the intensity peak toward the SW.

Figure 9 shows the ${ }^{12} \mathrm{CO}(1-0)$ isovelocity contours (first-moment map) superposed on the ${ }^{12} \mathrm{CO}(1-0)$ integrated 

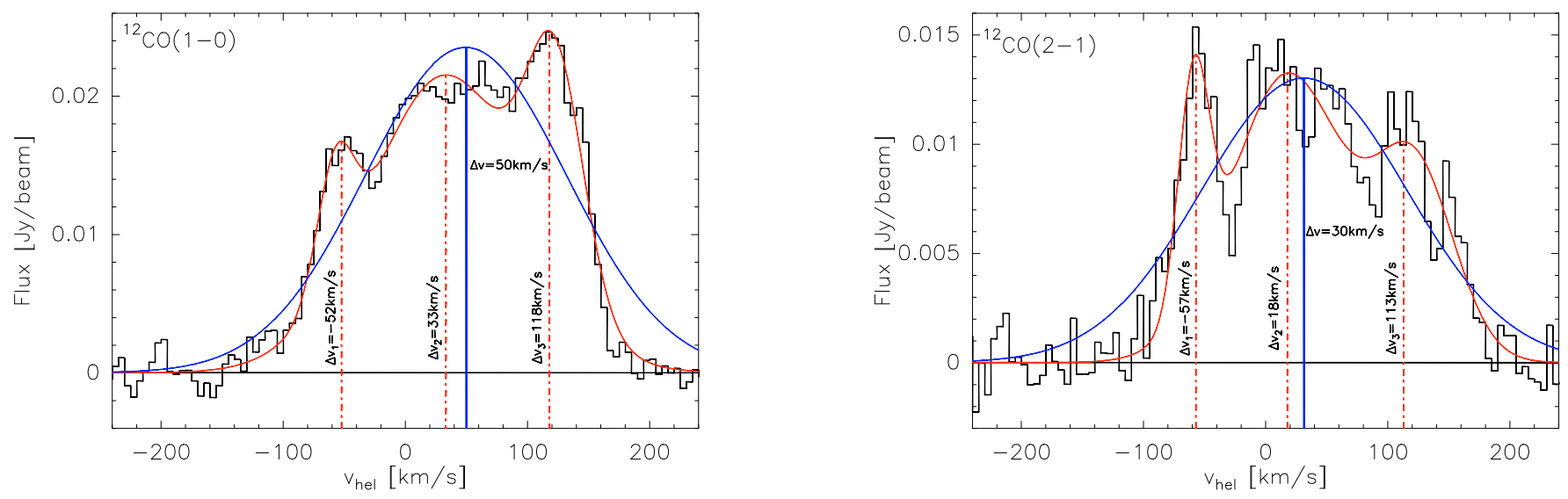

Fig. 6. Left panel: ${ }^{12} \mathrm{CO}(1-0)$ integrated spectrum, one component Gaussian (blue) fit, and three components Gaussian (red) fit in the inner $\sim 4^{\prime \prime}$ associated with the AGN source for PdBI $+30 \mathrm{~m}$ combined data. For the one component Gaussian fit the heliocentric systematic velocity is redshifted by $50 \mathrm{~km} \mathrm{~s}^{-1}$ with respect to the heliocentric velocity of the center $\left(0 \mathrm{~km} \mathrm{~s}^{-1}\right)$. Right panel: same for ${ }^{12} \mathrm{CO}(2-1)$. For the one component Gaussian fit the heliocentric systematic velocity is redshifted by $30 \mathrm{~km} \mathrm{~s}^{-1}$.
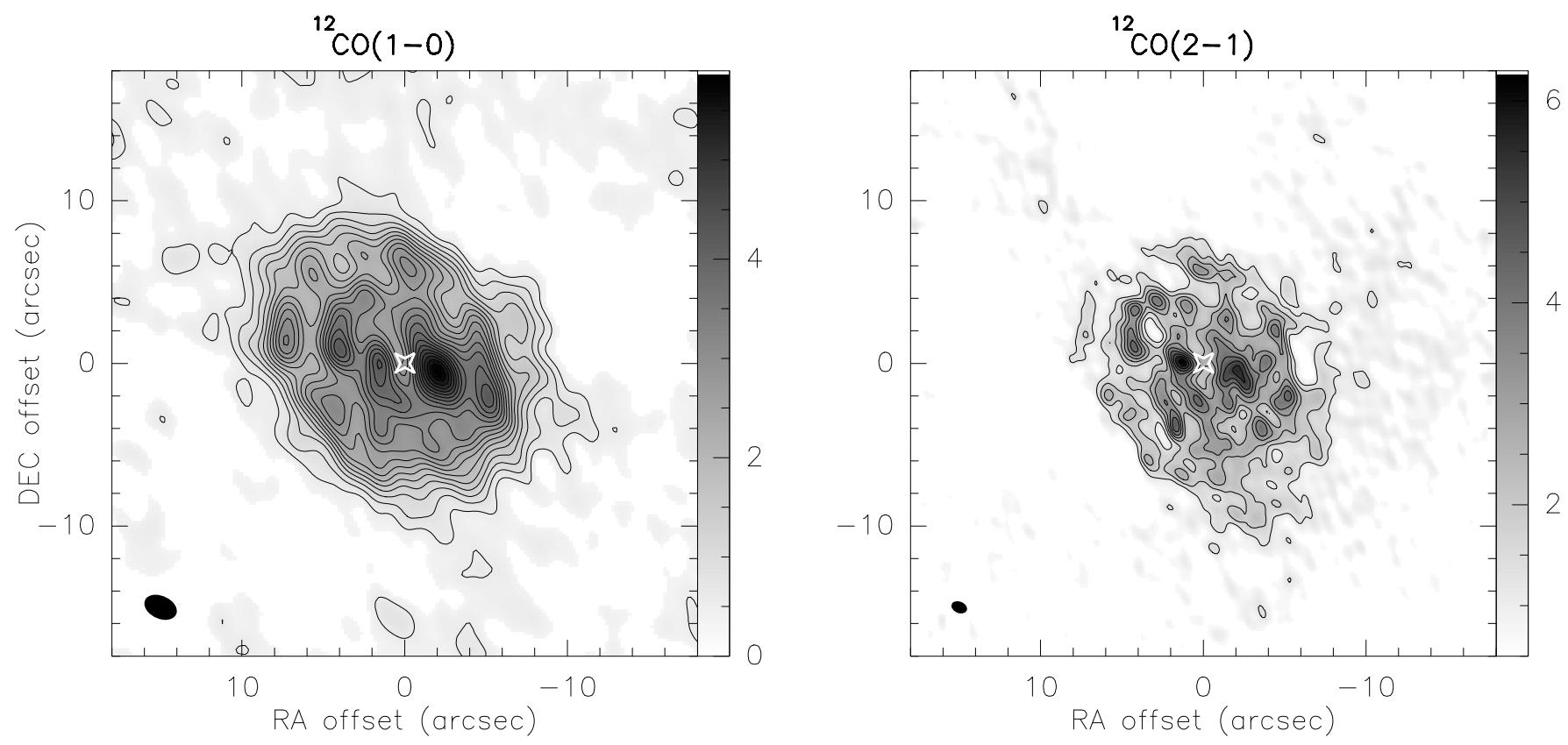

Fig. 7. Left panel: ${ }^{12} \mathrm{CO}(1-0)$ integrated intensity contours observed with the IRAM PdBI+30 m toward the center of NGC 5953. The white star marks the coordinates of the dynamical center of the galaxy, with offsets in arcseconds. The map, derived with $2 \sigma$ clipping, has not been corrected for primary beam attenuation. The rms noise level is $\sigma=0.09 \mathrm{Jy} \mathrm{beam}^{-1} \mathrm{~km} \mathrm{~s}^{-1}$ and contour levels run from $3 \sigma$ to $21 \sigma$ with $3 \sigma$ spacing. In this map the $\pm 130 \mathrm{~km} \mathrm{~s}^{-1}$ velocity range is used. The beam of $22^{\prime \prime} 1 \times 1{ }^{\prime \prime} 4$ is plotted in the lower left. Right panel: same for ${ }^{12} \mathrm{CO}(2-1)$. The rms noise level is $\sigma=0.2 \mathrm{Jy}_{\text {beam }}{ }^{-1} \mathrm{~km} \mathrm{~s}^{-1}$ and contour levels run from $3 \sigma$ to $10 \sigma$ with $3 \sigma$ spacing. The beam of $1{ }^{\prime \prime} 1 \times 0,7$ is plotted at lower left.

intensity. The white star indicates the dynamical center of the galaxy assumed coincident with the phase tracking center of our observations, and the velocities are relative to the systemic heliocentric velocity, $V_{\text {sys, hel }}=1990 \mathrm{~km} \mathrm{~s}^{-1}$. The dotted line traces the major axis of the galaxy determined from our observations $\left(\mathrm{PA}=45^{\circ} \pm 1^{\circ}\right)$, by maximizing the symmetry in the position velocity diagrams. This value is also the best-fit PA given by the elliptically-averaged surface brightness profiles discussed in Sect. 2.3.

Position-velocity $(\mathrm{p}-\mathrm{v})$ cuts along the major $\left(\mathrm{PA}=45^{\circ}\right)$ and minor axis $\left(\mathrm{PA}=135^{\circ}\right)$ of NGC 5953 are shown in Figs. 10 and 11 , respectively. In both figures, the ${ }^{12} \mathrm{CO}(1-0)$ emission is given in the top panel and the ${ }^{12} \mathrm{CO}(2-1)$ one in the bottom panel. The $\mathrm{p}-\mathrm{v}$ diagrams along the major axis reveal regular circular rotation and appear symmetric in the inner 10-11", both in ${ }^{12} \mathrm{CO}(1-0)$ and ${ }^{12} \mathrm{CO}(2-1)$. This regularity in the $\mathrm{p}-\mathrm{v}$ diagram of NGC 5953, more typical for normal spiral galaxies than interacting ones, has also been found in the ionized gas, including $\mathrm{H} \alpha$, [O III] (Gonzalez Delgado \& Perez 1996), and [N II] (Hernández-Toledo et al. 2003). The kinematics shown by the minor-axis $\mathrm{p}-\mathrm{v}$ diagrams (Fig. 11) are also quite regular, but show significant velocity dispersion for the ${ }^{12} \mathrm{CO}(1-0)$ close to the nucleus. This effect can be attributed to beam smearing.

\subsection{The rotation curve and dynamical mass}

We have derived a rotation curve (RC) from the p-v diagram taken along the kinematic major axis of NGC 5953 at a PA $=45^{\circ}$. 


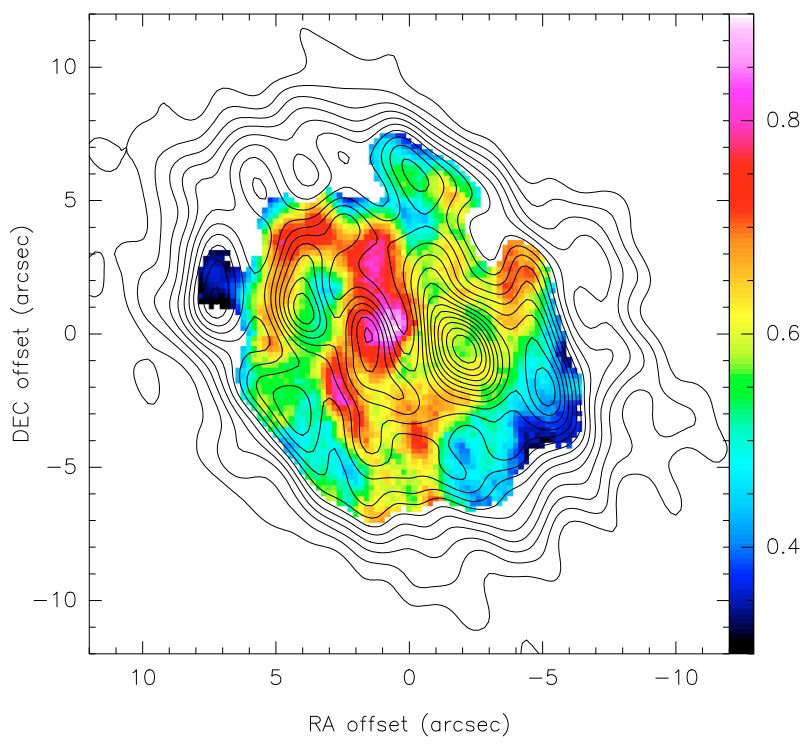

Fig. 8. Color scale of the $\mathrm{CO}(2-1) / \mathrm{CO}(1-0)$ ratio map and ${ }^{12} \mathrm{CO}(1-0)$ intensity map contours as in Fig. 7 (left panel).

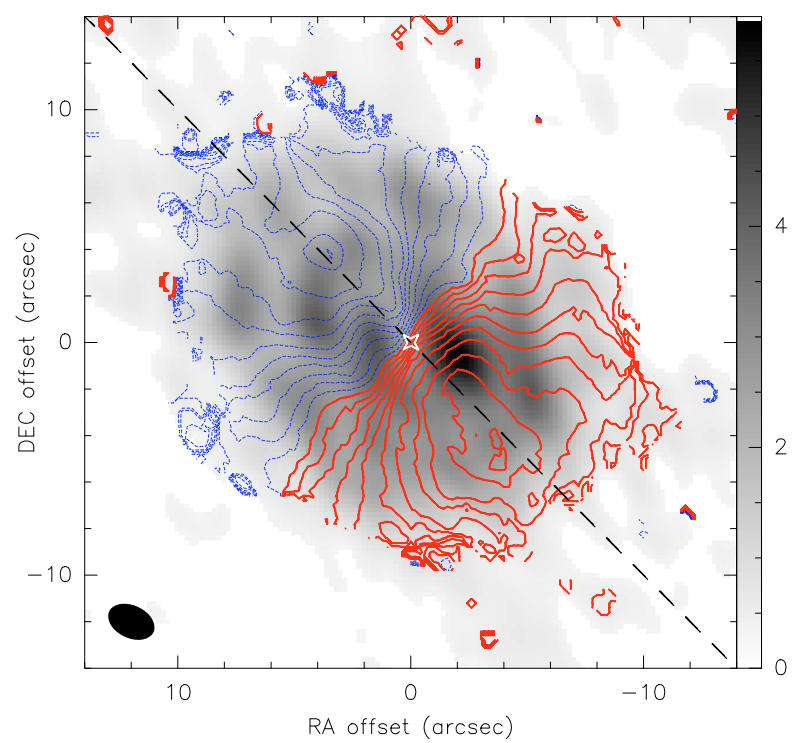

Fig. 9. Overlay of the integrated ${ }^{12} \mathrm{CO}(1-0)$ emission, same as Fig. 7 (left panel), with $\mathrm{CO}$ mean-velocity field in contours spanning the range -100 to $100 \mathrm{~km} \mathrm{~s}^{-1}$ in steps of $10 \mathrm{~km} \mathrm{~s}^{-1}$. The white star indicates the dynamical center of the galaxy. The velocities are referred to $V_{\text {sys,hel }}=$ $1990 \mathrm{~km} \mathrm{~s}^{-1}$, solid (red) lines are used for positive velocities, and dashed (blue) lines for negative velocities. The dotted line indicates the position angle of the major axis $\left(\mathrm{PA}=45^{\circ} \pm 1^{\circ}\right)$.

The terminal velocities were derived by fitting multiple Gaussian profiles to the spectra across the major axis. The fitted velocity centroids, corrected for $\sin i\left(i=42^{\circ}\right)$, give $V_{\text {obs }} / \sin i$ for each galactocentric distance.

For both lines the velocities (negative) to the northeast increase less steeply within $\sim 250 \mathrm{pc}$ than those to the south (positive velocities). Nevertheless, they converge to the same value at greater distances, so we averaged together the two curves derived from either side of the major axis. Because data for both lines were consistent, we combined both curves into an average by spline interpolation. The RCs from the two ${ }^{12} \mathrm{CO}$ transitions are shown in Fig. 12. They are consistent with those found for the
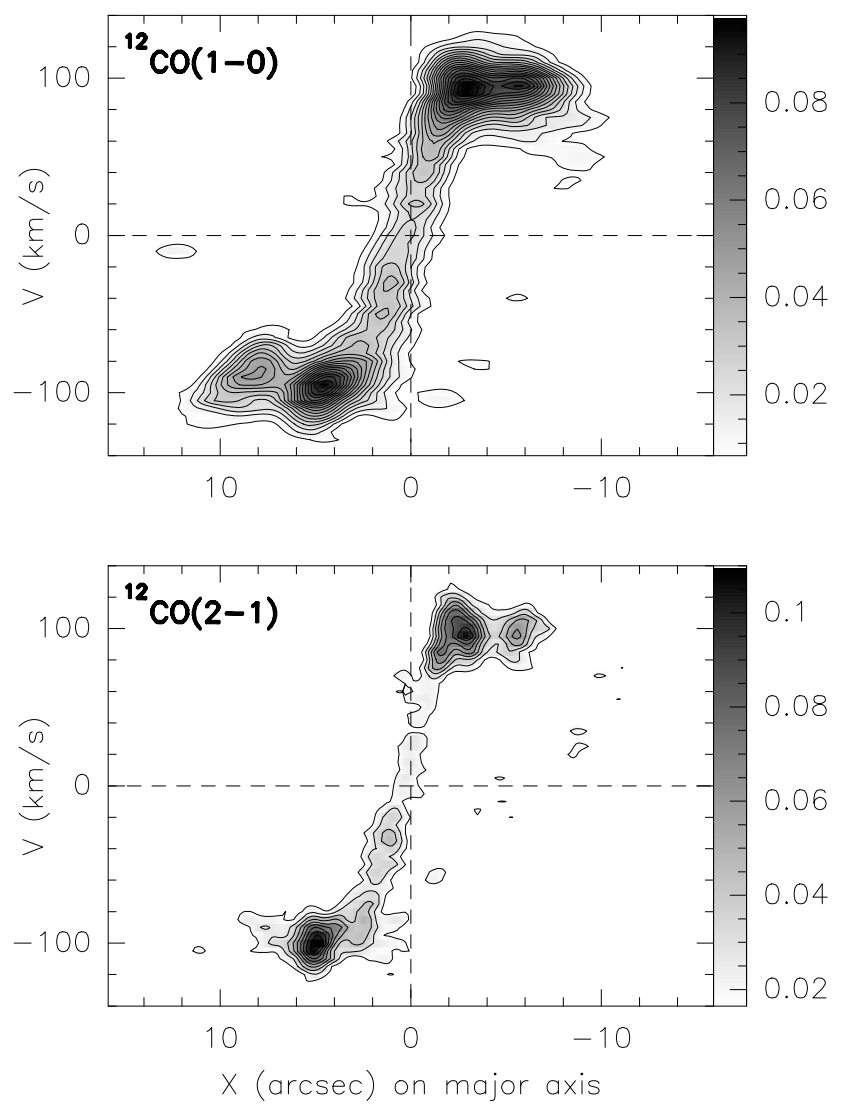

Fig. 10. Upper panel: ${ }^{12} \mathrm{CO}(1-0)$ position-velocity diagram along the major axis $\left(\mathrm{PA}=45^{\circ}\right)$ of NGC 5953 using the velocity range from -140 to $140 \mathrm{~km} \mathrm{~s}^{-1}$ contoured over a grey-scale representation. Contour levels are from $3 \sigma$ to $39 \sigma$ in steps of $2 \sigma\left(\sigma=2.5 \mathrm{mJy}^{-1}\right.$ beam $\left.^{-1}\right)$. The velocities are relative to $V_{\text {sys,hel }}\left(=1990 \mathrm{~km} \mathrm{~s}^{-1}\right)$ and $\mathrm{X}$ are the offsets along the major axis in arcsecs. Bottom panel: the same for ${ }^{12} \mathrm{CO}(2-1)$. Contour levels are from $3 \sigma$ to $20 \sigma$ in steps of $2 \sigma\left(\sigma=5.5 \mathrm{mJy}^{-1}\right.$ beam $\left.^{-1}\right)$.

ionized gas, as reported by Gonzalez Delgado \& Perez (1996) and Hernández-Toledo et al. (2003).

The ${ }^{12} \mathrm{CO}$ RCs are very regular and apparently this behavior is expected more for normal spiral galaxies than interacting systems. NGC 5953 and the companion are clearly interacting and their outer parts are perturbed as shown by the presence of a tidal tail seen both in optical and in HI emission (e.g., HernándezToledo et al. 2003; Haan et al. 2008). In NGC 5953, we are mapping the molecular gas in the inner parts that have a dynamical time-scale much shorter than the outer ones, and therefore they had the time to relax and reach an equilibrium state, perhaps after gas accretion/exchanges from/with NGC 5954. In other words, the regularity of the RCs in ${ }^{12} \mathrm{CO}$ is not unexpected.

In the major-axis $\mathrm{p}-\mathrm{v}$ diagrams, the velocity peaks at $\sim 120 /(\sin i) \mathrm{km} \mathrm{s}^{-1}$ at a radius of $\sim 2-4^{\prime \prime}(\sim 400 \mathrm{pc})$; however, the bulk of the gas is rotating at $\sim 98 /(\sin i) \mathrm{km} \mathrm{s}^{-1}$ (see Fig. 12). This is roughly consistent with what is found for the ionized gas, $\sim 115 /(\sin i) \mathrm{km} \mathrm{s}^{-1}$ by Hernández-Toledo et al. $(2003)^{5}$. The bulk velocity corrected for disk inclination, sin $i$ (we adopt $i=42^{\circ}$, see Sect. 4.1), becomes $\sim 146 \mathrm{~km} \mathrm{~s}^{-1}$. From the corrected bulk velocity we can estimate the dynamical mass within a certain radius using the formula $M(R)=\alpha \times R V^{2}(R) / G$, where $M(R)$ is in $M_{\odot}, R$ in $\mathrm{kpc}$, and $V$ in $\mathrm{km} \mathrm{s}^{-1}$. Assuming the most flattened disk-like distribution $(\alpha=0.6)$, the above formula

5 Taking into account their different adopted inclination. 

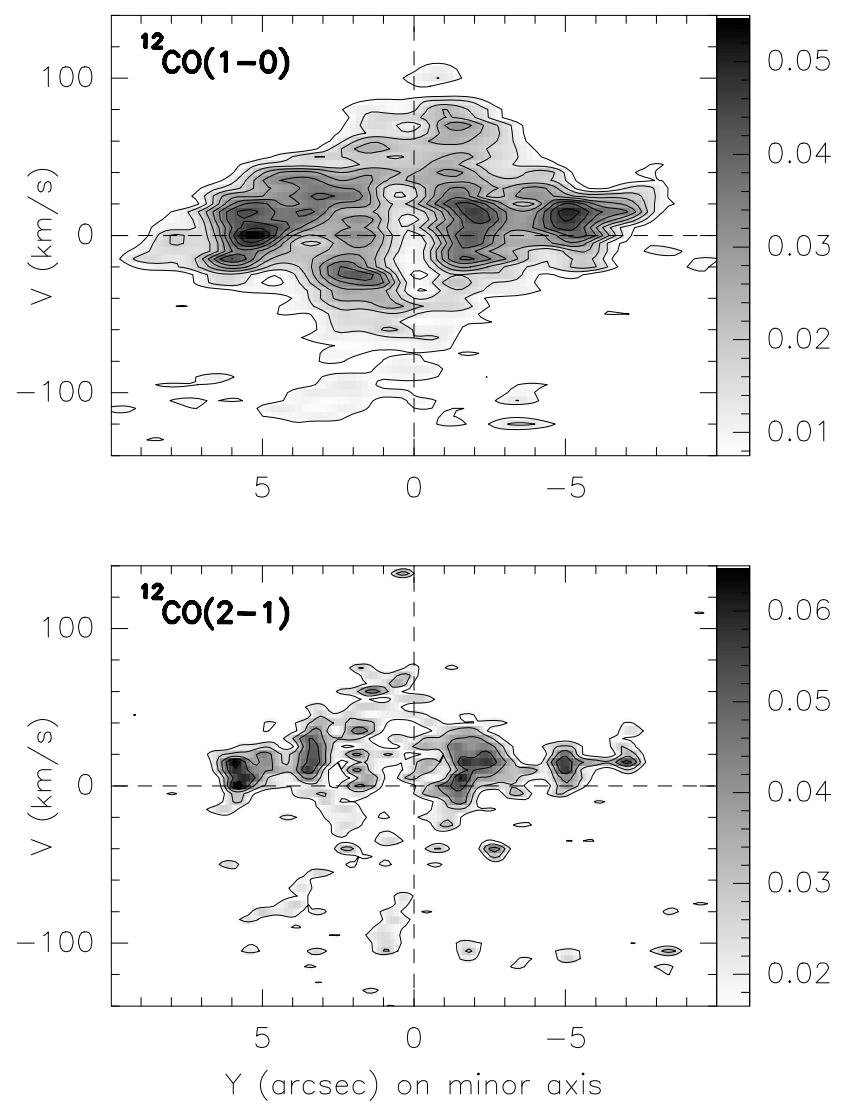

Fig. 11. Upper panel: same as Fig. 10 along the minor axis $\left(\mathrm{PA}=135^{\circ}\right)$ of NGC 5953. Contour levels are from $3 \sigma$ to $22 \sigma$ in steps of $2 \sigma . Y$ are the offsets along the minor axis in arcsecs. Bottom panel: the same for ${ }^{12} \mathrm{CO}(2-1)$. Contour levels are from $3 \sigma$ to $12 \sigma$ in steps of $2 \sigma$.

gives a dynamical mass of $M_{\text {dyn }}=2.4 \times 10^{9} M_{\odot}$ within a radius of $6^{\prime \prime}(\sim 0.8 \mathrm{kpc})$.

Assuming a roughly flat rotation curve, the dynamical mass should be $M_{\text {dyn }}=8.6 \times 10^{9} M_{\odot}$ within a radius of $21^{\prime \prime}(\sim 2.9 \mathrm{kpc})$. In the same region, we estimate a total molecular gas mass of $\sim 1.7 \times 10^{9} M_{\odot}$ (see Sect. 4.2), a fraction of $\sim 20 \%$ of the dynamical mass. Anyway, this percentage is subject to uncertainties. One of these could be due to the variation of the $\mathrm{H}_{2}-\mathrm{CO}$ conversion factor. In this work, as for similar studies of molecular gas, we assume a constant value for the $X$ conversion factor, for instance that determined for the Milky Way. However, the $X$ ratio is determined by various factors, such as the metallicity, the temperature, the cosmic ray density, and the UV radiation field (see Maloney \& Black 1988; Boselli et al. 2002), and its value can change by a factor 4-15 (e.g., Wilson 1995; Casasola et al. 2007). The $X$ value also changes with galaxy morphological type: usually galaxies earlier than Scd show values comparable to, or lower than, the Galactic one, while extremely late-type spirals or irregular galaxies tend to show higher values (Nakai \& Kuno 1995). Another possible uncertainty, but on the estimate of the dynamical mass, could be the suspected warp of the disk, discussed later in Sect. 5.3, that might give a wrong inclination of the disk, and so an incorrect rotation velocity.

\section{Dust, stellar structure, and star formation}

In this section we compare the ${ }^{12} \mathrm{CO}$ distribution of NGC 5953 with observations at other wavelengths. These comparisons have a dual aim. First, they allow the study of possible correlations between different tracers of the ISM, such as between molecular clouds, considered the birth site of future generations of stars, and the light from the stellar component. The second aim is to determine the location of dynamical resonances, which greatly aid the determination of gas flow in the circumnuclear regions of the disk. However, in the case of NGC 5953, we find no evidence for any non-asymmetric component which could drive resonances.

\subsection{Optical and near-infrared morphology}

Figure 13 (left panel) displays a HST/NICMOS/F606W band image of the inner $20^{\prime \prime}$ of NGC 5953, described in Sect. 2.3. We imposed that the brightness peak in this image coincides with the phase tracking center of our ${ }^{12} \mathrm{CO}$ observations and therefore with the dynamical center, as discussed in Sect. 4.1. The small peak at $\sim 3^{\prime \prime}$ west of center is almost certainly the foreground star identified in NGC 5953 by Rafanelli et al. (1990). Inspection of this figure shows a clearly-defined non-axisymmetric structure of $\sim 250 \mathrm{pc}$ in size, similar in shape and size to the [OIII] emission found by Gonzalez Delgado \& Perez (1996). A close-up of the inner 2" (Fig. 13, right panel) reveals an "S-shape" feature, perhaps associated with a nuclear bar (e.g., Hernández-Toledo et al. 2003). This bar-like signature is $\gtrsim 60 \mathrm{pc}$ in radius, with a PA $\sim 17^{\circ}$. Hernández-Toledo et al. (2003), by examining a contrast-enhanced $B$-band image of NGC 5953 (the same image as used here), identified this structure at about the same PA as the global major axis of the galaxy. We find (see Fig. 13, right panel) instead a more vertical orientation, PA $\sim 5-20^{\circ}$, depending on which features of the S-shape are used to define the PA. Rather than a nuclear bar, the S-shape structure could also be an optical counterpart of the radio jet (Krips et al. 2007a), because of the similar morphology, position angle, and size; this feature also seems to correspond to a higher excitation of the ionized gas (Gonzalez Delgado \& Perez 1996).

The inner $1.6 \mu \mathrm{m}$ morphology (HST/NICMOS/F160W) of NGC 5953 is shown in Fig. 14. The foreground star to the $3^{\prime \prime}$ to the west of the nucleus is clearly visible also at $1.6 \mu \mathrm{m}$. The flocculent spiral structure clearly visible in the optical (Fig. 13) is not seen in the NIR emission; there is also no NIR counterpart to the S-shape feature seen in the optical.

The morphology of the warm dust in the circumnuclear regions of NGC 5953 is shown in Fig. 15, where ${ }^{12} \mathrm{CO}(1-0)$ and ${ }^{12} \mathrm{CO}(2-1)$ intensities are contoured over the dust-only image described in Sect. 2.3. There is a dust emission peak to the SW, not exactly coincident with the ${ }^{12} \mathrm{CO}$ emission peak but roughly in the same direction. The bulk of the (putative) dust emission is configured in a smooth featureless disk, similar to that seen in the ${ }^{12} \mathrm{CO}$ emission.

\subsection{Stellar structure}

A large-scale view of the interacting companions NGC 5953 and NGC 5954 at $3.6 \mu \mathrm{m}$ is illustrated in Fig. 16. Like the $K$ band, this image traces the massive component of the stellar populations in galaxies, but at $3.6 \mu \mathrm{m}$ the extinction is about $1 / 3$ that of the $K$ band (Cardelli et al. 1989).

To investigate stellar structure, we performed a twodimensional B/D decomposition with galfit (Peng et al. 2002) on both the ground-based $H$-band image and IRAC $3.6 \mu \mathrm{m}$ image, fitting both galaxies (NGC 5953 and NGC 5954) simultaneously. In the fit, the models were convolved with the IRAC Point 


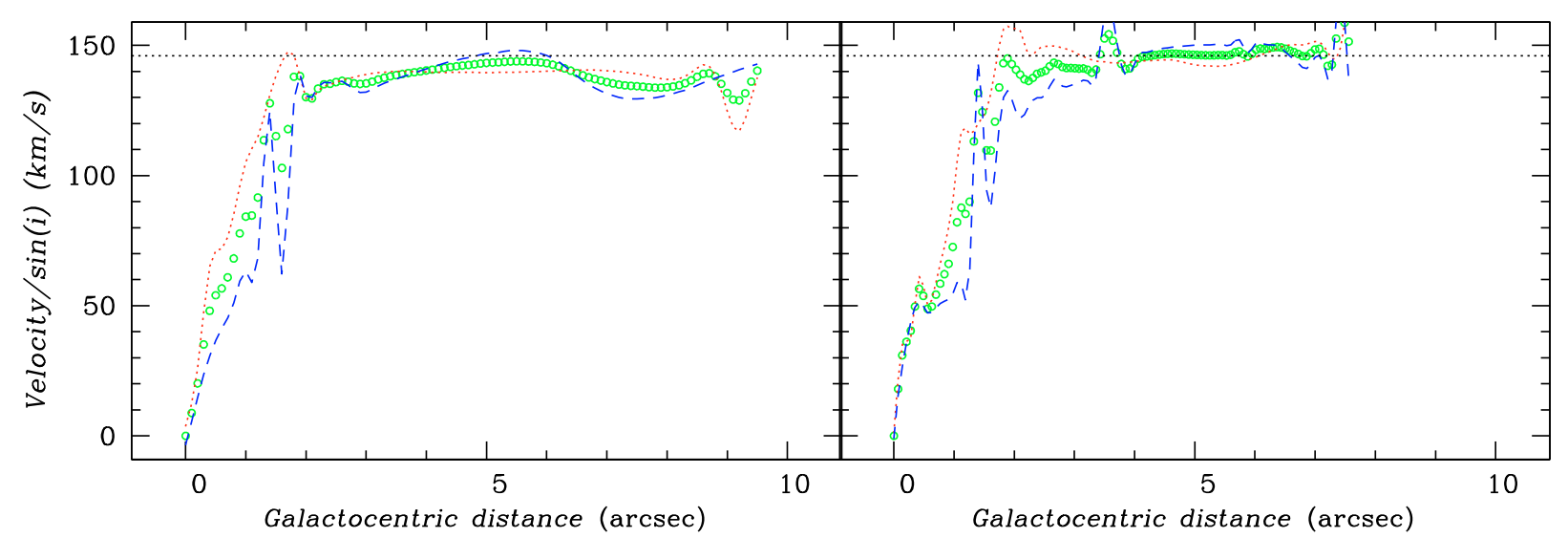

Fig. 12. Left panel: the ${ }^{12} \mathrm{CO}(1-0)$ rotation curve derived as described in the text. The positive (negative) velocities are shown as a red dotted (blue dashed) line; the open (green) circles show the average. The horizontal dotted line at $146 \mathrm{~km} \mathrm{~s}^{-1}$ indicates the velocity of the bulk of the molecular gas. Right panel: the same as the left panel, but for ${ }^{12} \mathrm{CO}(2-1)$.

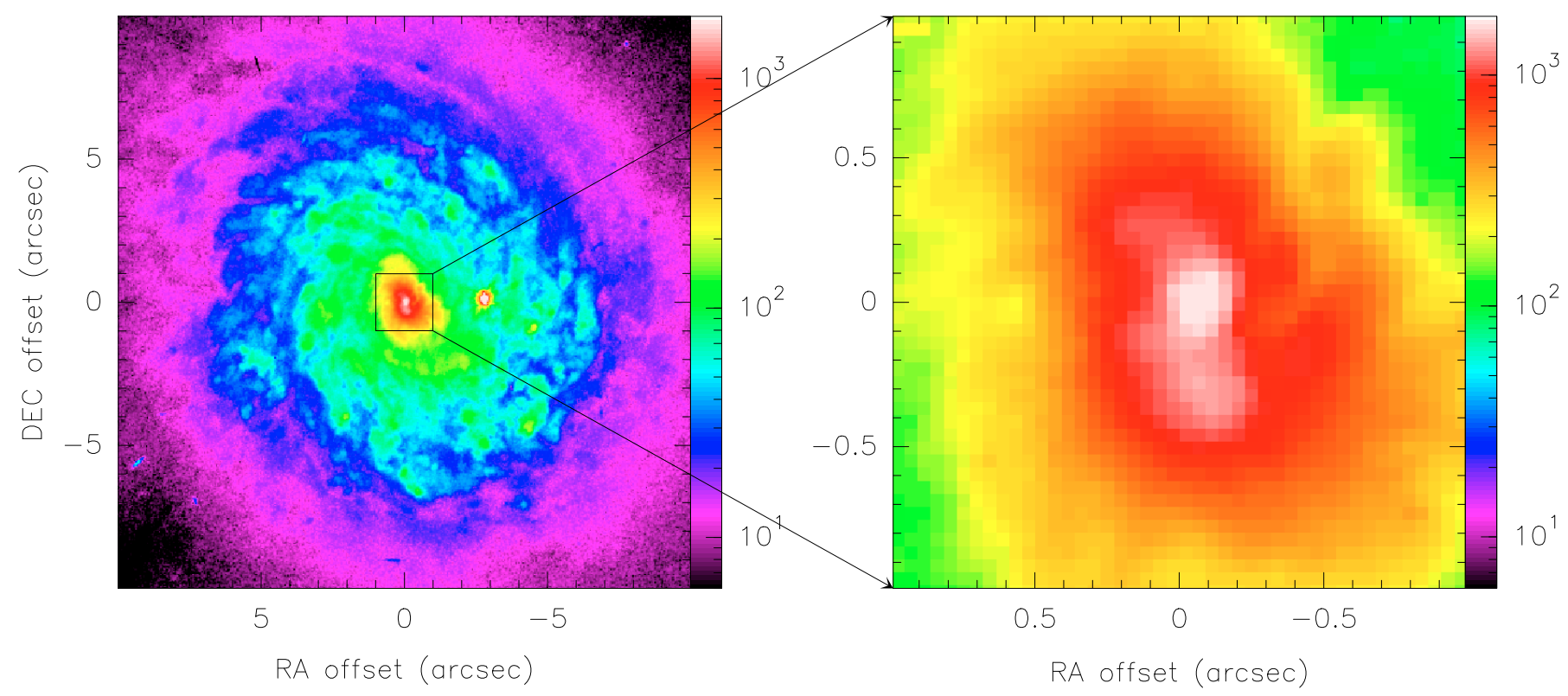

Fig. 13. Left panel: F606W band WFPC2/HST image of NGC 5953. The inner 20" are shown. Right panel: a zoom of the nuclear region. The inner $2^{\prime \prime}$ are shown.

Response Function (PRF) or an approximation of the groundbased Point Spread Function, as appropriate. The best fit included a Sersic generalized exponential bulge, an exponential disk, and a nuclear point source for NGC 5953, and a Sersic generalized exponential bulge (but with index $n \sim 1$, so this is probably in reality a disk) and a nuclear point source for NGC 5954. The IRAC fit has a reduced $\chi_{v}^{2}$ of 4.2 (averaged over NGC 5953 and NGC 5954), roughly ten times smaller than that in the $H$-band. Hence, in what follows, we mainly rely on the IRAC fit. The bulge comprises more than half $(\sim 59 \%)$ of the total $3.6 \mu \mathrm{m}$ light, consistently with its early Hubble type, but has a small $(n \sim 1)$ Sersic index, unlike most early-type bulges with $n \sim 3$ (Moriondo et al. 1998). The disk scalelength is $\sim 4 \mathrm{kpc}$, slightly larger than typical early-type spiral disks (e.g., Hunt et al. 2004); this could be due to the interaction and the proximity with NGC 5954.

The IRAC $3.6 \mu \mathrm{m}$ residuals from the $\mathrm{B} / \mathrm{D}$ decomposition are shown in Fig. 17, with the ${ }^{12} \mathrm{CO}(1-0)$ (left panel) and the ${ }^{12} \mathrm{CO}(2-1)$ (right panel) in contours. The residuals show a low-amplitude circumnuclear "ring" with a radius of $\sim 5-7$ ". The ${ }^{12} \mathrm{CO}(1-0)$ and ${ }^{12} \mathrm{CO}(2-1)$ disks nearly coincide in size with the ring structure in the residuals.

Because the size of the ring in the IRAC residuals is typical of small-scale "doughnuts" associated with the diffraction limit and PRF incompatibilities, we wanted to verify the reality of the ring-like feature seen in the $3.6 \mu \mathrm{m}$ residuals. If real, it should also appear in the $H$-band residuals, which are shown in the left panel of Fig. 18. Inspection of the figure shows that the ring is indeed present, delineated by a series of surface brightness excesses. The intensity contrast in these is around a factor of 10 relative to the regions external to the ring. The presence of the ring in both sets of residuals suggests that it is a real feature, rather than an artefact of IRAC PRF mismatching. Moreover, intensity peaks in the ring-like structure are also present in the un-sharp masked HST/NICMOS/F160W map (see Hunt \& Malkan 2004). We therefore conclude that the ring in the NIR residuals is real, and probably stellar in nature, as we discuss below. 


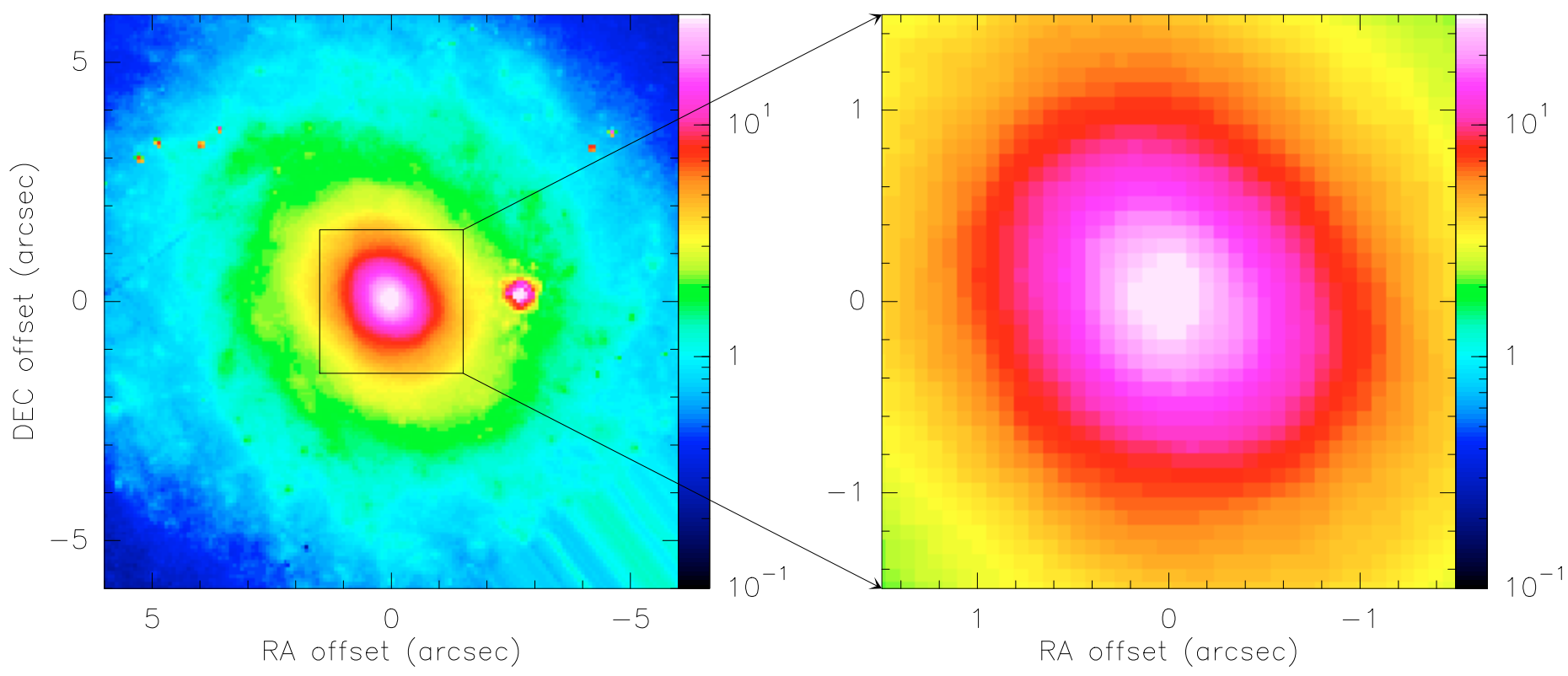

Fig. 14. Left panel: F160W band NICMOS/HST image of NGC 5953. The inner 12" are shown. Right panel: a zoom of the nuclear region. The inner $3^{\prime \prime}$ are shown.

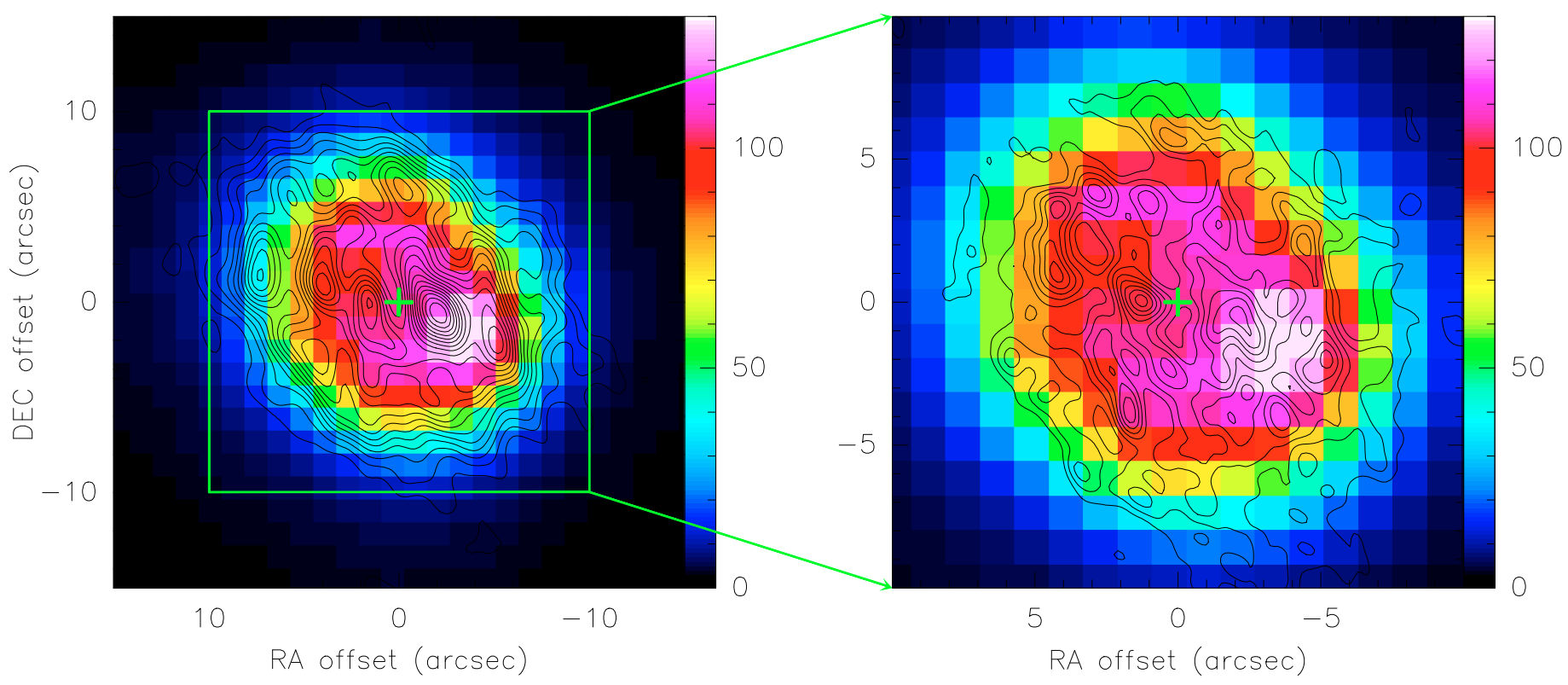

Fig. 15. Left panel: ${ }^{12} \mathrm{CO}(1-0)$ integrated intensity contours as in Fig. 7 (left panel) overlaid on the IRAC $8.0 \mu$ m "dust-only" image of NGC 5953 in false color. The (green) cross marks the dynamical center. The central $30^{\prime \prime}$ are shown. Right panel: same for ${ }^{12} \mathrm{CO}(2-1)$ integrated intensity contours as in Fig. 7 (right panel). The central 20" are shown.

\subsection{The stellar ring}

The right panel of Fig. 18 displays the ARNICA/NOT $J-K$ color image of NGC 5953. The nucleus of NGC 5953 and a small region to the west have extremely blue $J-K$ colors $(\sim 0.7$ and $\sim 0.3$, respectively), while the disk has rather normal ones $(\sim 1.0)$. The small knot $3^{\prime \prime}$ west of center, visible also in the WFPC2/F606W (Fig. 13) and NICMOS/F160W (Fig. 14) images, coincides almost certainly with the star identified by Rafanelli et al. (1990). There may be a few isolated regions $\left(\sim 1^{\prime \prime}\right)$ to the east of the nucleus with a red excess $(J-K \sim 1.3)$, but there is no evidence in the $J-K$ colors for the ring seen in the $B / D$ residuals. This suggests that the excess residual ring is a stellar ring, with colors similar to the surrounding disk.
A hint of the circumnuclear ring in NGC 5953 also appears in previous optical observations of the ionized gas. With the integral-field spectrograph SAURON operating on the William Herschel Telescope, Falcón-Barroso et al. (2006) found recent star formation in a circumnuclear ring with a radius of $\sim 6-$ $7^{\prime \prime}$. The $\mathrm{H} \beta$ emission is apparently distributed in a circumnuclear disk, roughly coincident with the $\mathrm{CO}$ one, but the $\mathrm{H} \beta$ velocity dispersion and the $[\mathrm{OIII}] / \mathrm{H} \beta$ ratio are lower in a ring-like structure than in the main circumnuclear disk. This could be because the nucleus excites gas in an extended region (Falcón-Barroso et al. 2006), or be due to a true ring in the dynamical sense of a resonance. Lower velocity dispersion and lower $[\mathrm{OIII}] / \mathrm{H} \beta$ are both associated with enhanced star formation, leading Hernández-Toledo et al. (2003) to conclude that 


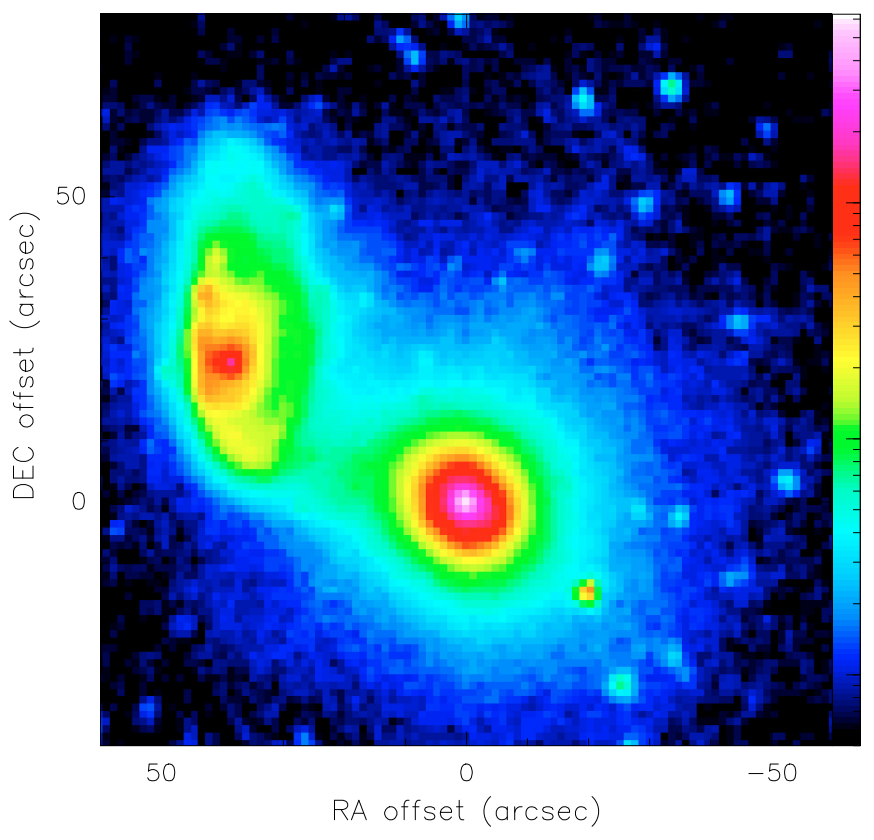

Fig. 16. IRAC $3.6 \mu \mathrm{m}$ image of NGC 5953 and NGC 5954, coordinate system centered on NGC 5953 (with N up, E left). The inner 120" are shown.

NGC 5953 hosts a circumnuclear ring. The size of this starforming ring is coincident with that of the NIR ring in the residuals. Figure 19 shows the superposition of the $3.6 \mu \mathrm{m}$ residuals image (Fig. 17, left panel), represented in (green) contours, on the $H$-band residuals image (Fig. 18, left panel). This figure clearly shows the coincidence in size between the two NIR rings, the same size of the star-forming ring seen in the ionized gas.

Because the ring is visible in ionized gas, but in the NIR appears as stars, with no sign of hot dust because of normal $J-K$ colors, we interpret it as an ongoing starburst, with a stellar Red Super Giant (RSG) population. This would imply that the ring feature is at least 10-15 Myr old, because RSGs onset at roughly that time (Leitherer et al. 1999). However, the presence of hydrogen recombination lines (e.g., Gonzalez Delgado \& Perez 1996; Hernández-Toledo et al. 2003) means that star formation is still ongoing, although the exact age is difficult to determine (5-10 Myr).

In addition to the star-forming+stellar ring, the observations of Falcón-Barroso et al. (2006) suggest that the stars in the outer regions of the circumnuclear disk are counter-rotating relative to stars in the inner disk and the ionized and molecular gas. In this (putative) kinematically decoupled component (KDC), the stars in the outer parts are redshifted toward the NE, while the ionized+molecular gas and the stars in the inner parts are redshifted toward the SW. The separation between the two sets of kinematics is at roughly $7-8^{\prime \prime}$ radius, so the ring could be the separation between the two regimes. The most likely explanation for this, as proposed by Falcón-Barroso et al. (2006), is that in NGC 5953 we are witnessing the formation of a decoupled component as a result of an ongoing interaction; the gas in the inner parts could have been accreted from the companion NGC 5954. The KDC could be in its early stages, as judged by our stellar population age estimate of $\gtrsim 10-15$ Myr. This age would be consistent with the onset of the interaction of $40 \mathrm{Myr}$ ago, as suggested by Jenkins (1984); this age from the time of closest approach is almost independent of the details of the model. This kind of counter-rotation has been also studied with simulations (e.g., di Matteo et al. 2008), and the presence of the HI tidal tail gives some hint that the interaction in the pair NGC 5953/5954 is prograde, i.e., that at least one of the disks involved has its spin axis parallel to the orbital velocity of the merger (Hibbard \& van Gorkom 1996; Iono et al. 2005).

Alternatively, the observed counter-rotation could be only apparent and not real, due to a warped disk in the center. The inner disk is not highly inclined, and a small tilt of the plane starting at a radius close to that of the ring, is able, because of projection effects, to reverse the apparent sense of rotation, without the presence of a true counter-rotation. A warped inner disk is expected from the close tidal interaction between NGC 5953 with NGC 5954, and the obvious tidal tails in optical and HI. The warped disk hypothesis is supported by the fact that the ionised gas also, and not only the stars, shows some hint of counter-rotation. The existence of two gas flows counterrotating in the same disk, is a very unlikely situation, that could be at best transient. The gas counter-rotation just outside the ring is visible in the Sauron velocity maps of Falcón-Barroso et al. (2006). Indeed, the velocity maps in $\mathrm{H} \beta$, and even more the [OIII] lines reveal on the NE part of the major axis that the velocity from blueshifted turns to redshifted again, by an amount of $\sim 40 \mathrm{~km} \mathrm{~s}^{-1}$. The apparent counter-rotation might look more striking on the stellar velocity maps, only because the stellar component is less rotation-dominated in the inner disk, because of the higher velocity dispersion. So the velocities on the major axis turn from $-40 \mathrm{~km} \mathrm{~s}^{-1}$ to $40 \mathrm{~km} \mathrm{~s}^{-1}$, while the gas is turning from $-100 \mathrm{~km} \mathrm{~s}^{-1}$ to $40 \mathrm{~km} \mathrm{~s}^{-1}$. But it is clear that in the NE part of the major axis, the stars and gas are co-rotating at the same velocities, and apparently counter-rotate relative to the inner disk. Since the gas cannot be counter-rotating with itself for a long time, the interpretation of a warp in the outer disk is probably more realistic. However, if the gas is actually counter-rotating at a radius of $1.6 \mathrm{kpc}$, then it could arise from external accretion at that radius. This gas is configured in a full gaseous disk, of about $8 \mathrm{kpc}$ radius, as mapped in HI by Chengalur et al. (1994). We then can only witness a transient phase of settling of the accreted gas, which will soon align its rotation with the main disk, rotating in the same sense as the $\mathrm{CO}$ gas and stars in the center.

\subsection{Star formation in NGC 5953}

Most of the star formation in NGC 5953 occurs in the circumnuclear region sampled by our ${ }^{12} \mathrm{CO}$ observations. The starformation rate (SFR) given by $\mathrm{H} \beta$ within a FOV of $33^{\prime \prime} \times 41^{\prime \prime}$ is $0.6 M_{\odot} \mathrm{yr}^{-1}$ (Falcón-Barroso et al. 2006). This corresponds to $\sim 0.024 M_{\odot} \mathrm{yr}^{-1} \mathrm{kpc}^{-2}$. We measure a molecular mass of $1.7 \times 10^{9} M_{\odot}$ in a roughly similar region (see Sect. 4.2), which would give a molecular surface density of $\sim 60 M_{\odot} \mathrm{pc}^{-2}$. In a Kennicutt-Schmidt (KS) law diagram without the neutral atomic gas component, this would place NGC 5953 in the transition region between normal spiral disks and circumnuclear starbursts (Kennicutt 1998). In fact, HI observations show significant streaming motions over the inner $17 \mathrm{kpc}$ inconsistent with global rotation (Iono et al. 2005; Haan et al. 2008). Hence, the HI probably does not take part in the star formation activity in this region. It is probable that the enhanced star formation in NGC 5953 is caused by dynamical perturbations induced by the interaction.

In NGC 5953, the high $\mathrm{H}_{2}$ surface density and the low SFR imply that the distribution of molecular gas (traced by $\mathrm{CO}$ ) does not correlate locally with the SFR; the large reservoir of molecular gas is not converted into stars proportionally to the KS law 


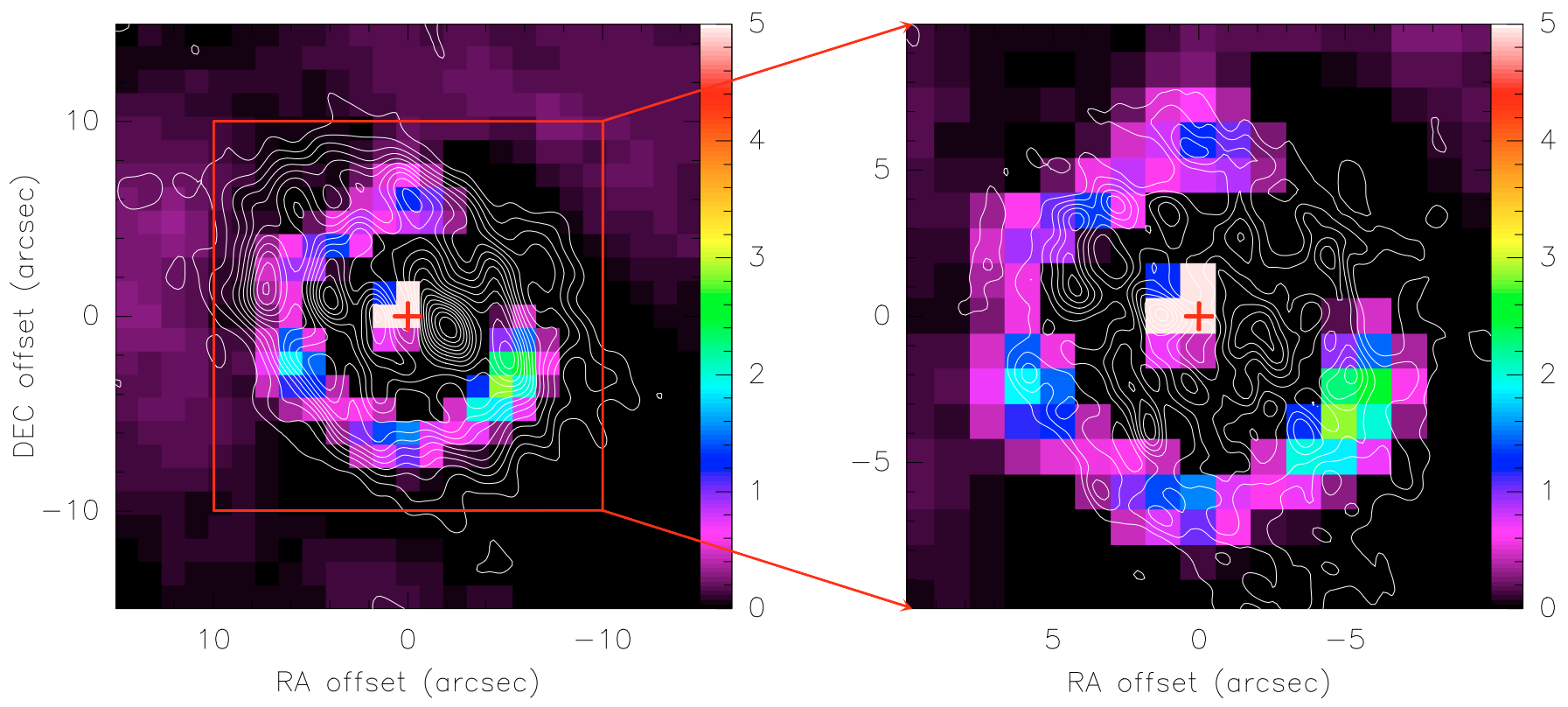

Fig. 17. Left panel: ${ }^{12} \mathrm{CO}(1-0)$ integrated intensity contours as in Fig. 7 (left panel) overlaid on the residuals (in MJy sr ${ }^{-1}$ ) of the B/D decomposition performed on the IRAC $3.6 \mu \mathrm{m}$ image. The (red) cross marks the dynamical center. The central $30^{\prime \prime}$ are shown. Right panel: same for ${ }^{12} \mathrm{CO}(2-1)$ integrated intensity contours as in Fig. 7 (right panel). The central $20^{\prime \prime}$ are shown.
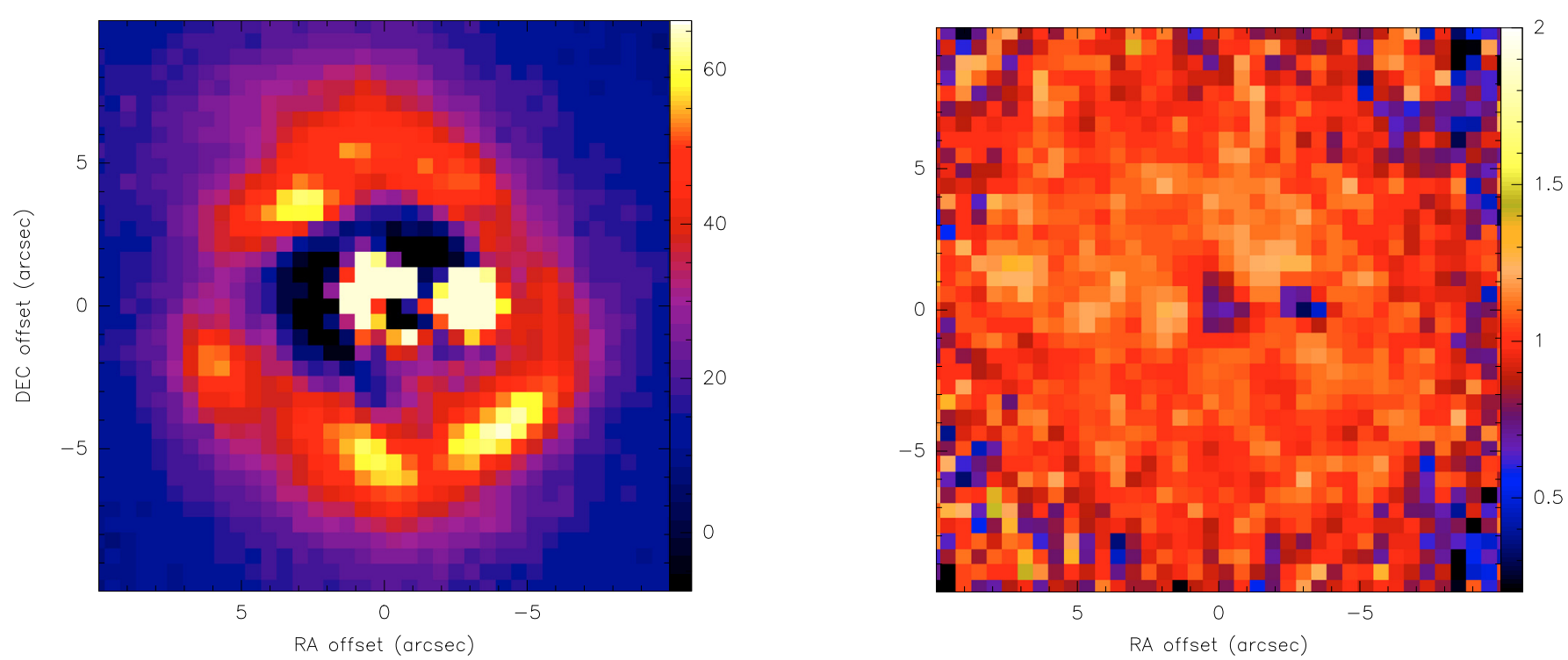

Fig. 18. Left panel: $H$-band residuals from the galfit B/D decomposition. Right panel: the $J-K$ color image. The FOV for both panels is $20^{\prime \prime}$ with north up, and east to the left. In both images, the foreground star $\sim 2$ '. 7 to the west is evident.

on small spatial scales. At present, the non-local correlation between SFR and gas is highly debated (Bigiel et al. 2008; Leroy et al. 2008). Other molecular transitions could be likely better indicators of SFR than the total $\mathrm{H}_{2}$ content traced by CO. Tracers of dense molecular gas, such as $\mathrm{HCN}(1-0)$ and $\mathrm{HCO}^{+}(1-0)$ lines, are suspected to better correlate with the SFR (e.g., Gao \& Solomon 2004a,b; Wu et al. 2005; Graciá-Carpio et al. 2008).

\section{Gravitational torques on the molecular gas}

In this section we study whether gravitational torques, derived from the stellar potential in the inner region of NGC 5953, can account for the gas kinematics derived from $\mathrm{CO}$ and examine the efficiency of gravitational torques exerted on the gas. As described in previous NUGA papers (e.g., García-Burillo et al. 2005), we assume that NIR images give the best approximation for the total stellar mass distribution, being less affected than optical images by dust extinction or stellar population bias.

\subsection{Evaluation of the gravitational potential}

We computed the torques using Spitzer/IRAC and ARNICA images. They both yield comparable results, but we use in the following only the NOT/ARNICA images which give more spatial resolution. We perform the subtraction of foreground stars, deprojection, and resampling, as described in other NUGA papers (e.g., García-Burillo et al. 2005). 


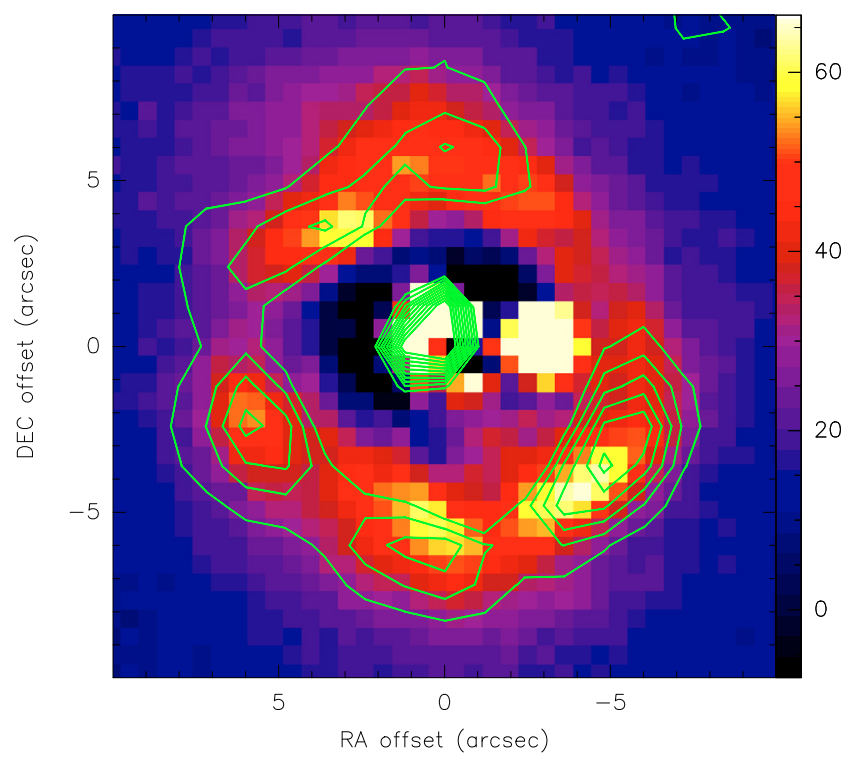

Fig. 19. 3.6 $\mu \mathrm{m}$ residuals in (green) contours (Fig. 17) overlaid on the $H$-band residuals (Fig. 18, left panel). The inner $20^{\prime \prime}$ are shown.

We repeat here some definitions and assumptions used to evaluate the gravitational torques. The NIR image is completed in the vertical dimension by assuming an isothermal plane model with a constant scale height, equal to $\sim 1 / 12$ th of the radial scalelength of the image. The potential is derived by a Fourier transform method and assuming a constant mass-to-light $(\mathrm{M} / \mathrm{L})$ ratio whose value is chosen to better reproduce the observed ${ }^{12} \mathrm{CO}$ rotation curve. Beyond a radius of $20^{\prime \prime}$ (or $5.4 \mathrm{kpc}$ in diameter), the mass density is set to 0 , thus suppressing any spurious $m=4$ terms. This assumption is sufficient to compute the potential over the PdBI ${ }^{12} \mathrm{CO}(1-0)$ primary beam.

To characterize the non-axisymmetry of the potential, we expand its $\Phi(R, \theta)$ in Fourier components (m-modes), following Combes \& Sanders (1981):

$\Phi(R, \theta)=\Phi_{0}(R)+\sum_{m=1}^{\infty} \Phi_{m}(R) \cos \left[m \theta-\phi_{m}(R)\right]$

where $\Phi_{m}(R)$ and $\phi_{m}(R)$ are the amplitude and phase of the $m$ mode, respectively.

The strength of each $m$-Fourier component, $Q_{m}(R)$, is defined by the ratio between tangential and radial forces, $Q_{m}(R)=$ $m \Phi_{m} / R\left|F_{0}(R)\right|$. The strength of the total non-axisymmetric perturbation is defined by:

$Q_{\mathrm{T}}(R)=\frac{F_{\mathrm{T}}^{\max }(R)}{F_{0}(R)}$

where $F_{\mathrm{T}}^{\max }(R)$ and $F_{0}(R)$ represent the maximum amplitude of the tangential force and the mean axisymmetric radial force, respectively. Figures 20 show the strengths and phases vs. radius for the first $m$ components. There is only a weak $m=2$ perturbation; the strongest one is $m=1$.

\subsection{Evaluation of gravity torques}

From the derivatives of $\Phi(R, \theta)$ on each pixel we obtain the forces per unit mass $\left(F_{x}\right.$ and $\left.F_{y}\right)$ and therefore the torques per unit mass $t(x, y)$ can be computed by:

$t(x, y)=x F_{y}-y F_{x}$.
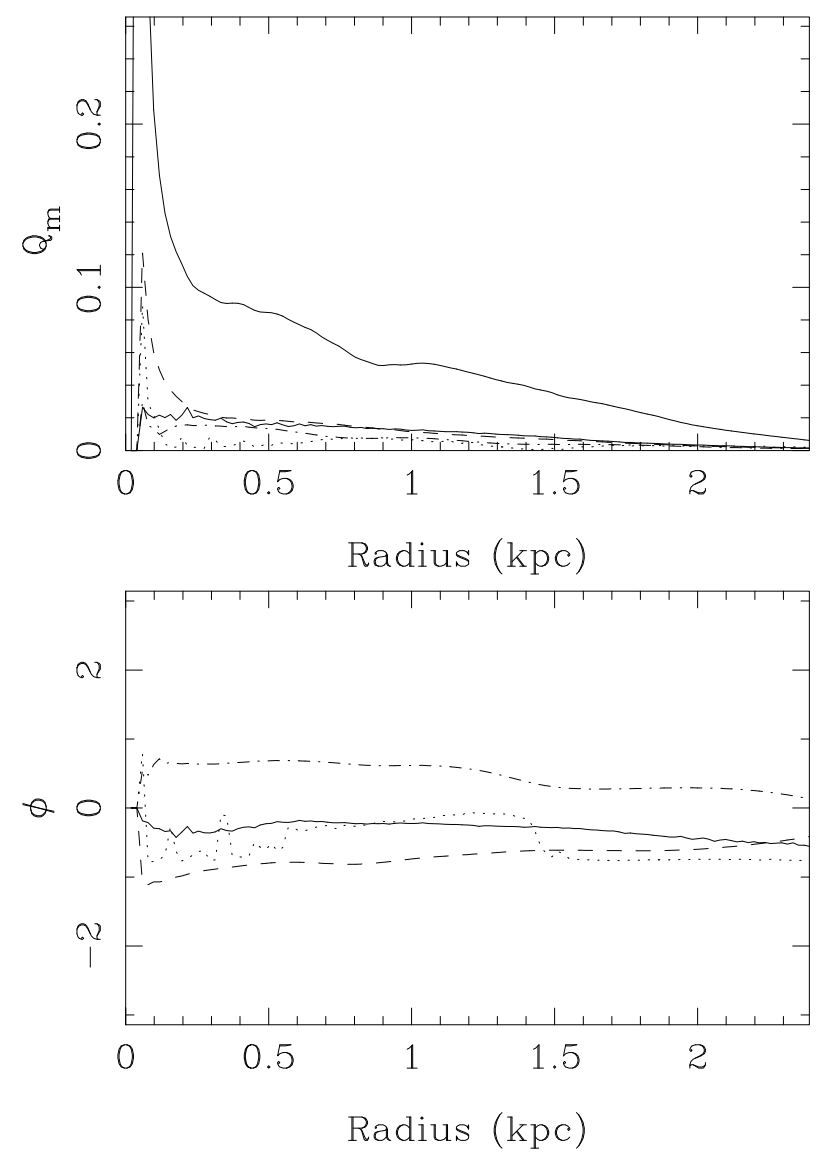

Fig. 20. The strength $Q$ (top) and phase $\Phi$ (bottom) of the $m=1,2,3,4$ Fourier components of the potential, derived from the NIR image. The full lines correspond to $m=2$ and the total strength, the dashed line to $m=1$, dot-dash to $m=3$, and dots to $m=4$.

The torque map is oriented according to the sense of rotation in the plane of the galaxy, and the combination of the torque map and the gas density $\Sigma$ map allows us to derive the net effect on the gas at each radius. The gravitational torque map weighted by the gas surface density $t(x, y) \times \Sigma(x, y)$, normalized to its maximum value, is shown in Figs. 21 and 22 for ${ }^{12} \mathrm{CO}(1-0)$ and ${ }^{12} \mathrm{CO}(2-$ $1)$, respectively. The observed gas distribution is representative of the time spent by a molecular cloud on a typical orbit at this location.

To estimate the efficiency of the radial gas flow induced by the torques, we first compute the torque per unit mass averaged over azimuth, using $\Sigma(x, y)$ as the actual weighting function:

$t(R)=\frac{\int_{\theta} \Sigma(x, y) \times\left(x F_{y}-y F_{x}\right)}{\int_{\theta} \Sigma(x, y)}$

where $t(R)$ is, for definition, the time derivative of the specific angular momentum $L$ of the gas averaged azimuthally, $t(R)=$ $\mathrm{d} L /\left.\mathrm{d} t\right|_{\theta}$. Now to have dimensionless quantities, we normalize this variation of angular momentum per unit time to the angular momentum at this radius and to the rotation period. We then estimate the efficiency of the gas flow as the average fraction of the gas specific angular momentum transferred in one rotation $\left(T_{\text {rot }}\right)$ by the stellar potential, as a function of radius:

$\frac{\Delta L}{L}=\left.\frac{\mathrm{d} L}{\mathrm{~d} t}\right|_{\theta} \times\left.\frac{1}{L}\right|_{\theta} \times T_{\text {rot }}=\frac{t(R)}{L_{\theta}} \times T_{\text {rot }}$

where $L_{\theta}$ is assumed to be well represented by its axisymmetric estimate, $L_{\theta}=R \times v_{\text {rot }}$. Figures 23 show $\Delta L / L$ curves for 


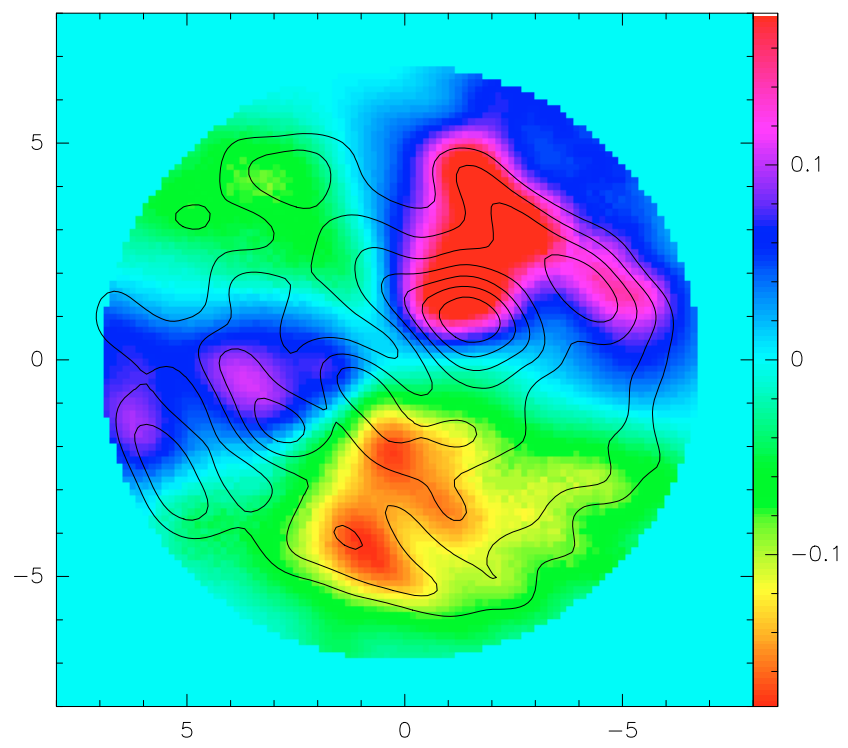

Fig. 21. The ${ }^{12} \mathrm{CO}(1-0)$ contours are overlaid onto the gravitational torque map $(t(x, y) \times \Sigma(x, y)$, as defined in text) in the center of NGC 5953. The torque map (color scale) is plotted on a symmetric palette (wedge) although the maximum positive torque is $50 \%$ higher in absolute value then the negative torque. The map is deprojected, and rotated so that the major axis of the galaxy is oriented parallel to the abscissa $\mathrm{O} x$.

NGC 5953 derived from the ${ }^{12} \mathrm{CO}(1-0)$ (left) and the ${ }^{12} \mathrm{CO}(2-1)$ (right) data. The (red) dashed area corresponds to the resolution limit of our observations. In the left panel, the $\mathrm{CO}(1-0)$ map resolution $(\sim 120 \mathrm{pc})$ is the limiting factor, while, on the right, the $\mathrm{CO}(2-1)$ map resolution $(\sim 60 \mathrm{pc})$ and the similar NIR image resolution $(\sim 70 \mathrm{pc})$ are the constraining factors. This figure shows that the torques are weak and predominantly positive between $\sim 100-400 \mathrm{pc}$ in both ${ }^{12} \mathrm{CO}(1-0)$ and ${ }^{12} \mathrm{CO}(2-1)$. Nothing can be said for the negative torques inside a radius of $\sim 120 \mathrm{pc}$ for ${ }^{12} \mathrm{CO}(1-0)$ and $\sim 70 \mathrm{pc}$, below our resolution. In ${ }^{12} \mathrm{CO}(2-1)$ the torques are noisier than in ${ }^{12} \mathrm{CO}(1-0)$, maybe because of a more clumpy emission.

In summary, torques are predominantly positive in the region $\sim 100-400$ pc for both lines, and the gas is not apparently fueling the central $\sim 100 \mathrm{pc}$ region down to the effective spatial resolution of our observations. In addition, the absolute value of the torques is very small, less than $5 \%$ of the gas angular momentum is exchanged in each rotation. This is due to the very regular and almost axisymmetric total mass and gas distributions in the center of the galaxy.

\section{Summary and conclusions}

The molecular gas in the Seyfert 2/LINER galaxy NGC 5953 has been mapped with high resolution $\left(22^{\prime \prime} 1 \times 11^{\prime \prime} .4\right.$ for the ${ }^{12} \mathrm{CO}(1-$ $0)$ line and $1^{\prime \prime} .1 \times 0.7$ for the ${ }^{12} \mathrm{CO}(2-1)$ line) inside a radius of $\sim 20^{\prime \prime}(\sim 2.7 \mathrm{kpc})$. The ${ }^{12} \mathrm{CO}$ emission is distributed over a disk of diameter of $\sim 16^{\prime \prime}$ with several peaks randomly distributed. The strongest intensity peak is not located in the center of the galaxy but shifted $\sim 2-3^{\prime \prime}$ toward the west/southwest. The kinematics of the molecular gas show a general regularity with some local wiggles especially to the west of the nucleus maybe associated with the intensity peak toward the SW.

By comparing the molecular gas distribution with observations at other wavelengths, we studied correlations between different tracers of the ISM. Optical and NIR morphology has been studied by analyzing different images. The F606W/HST

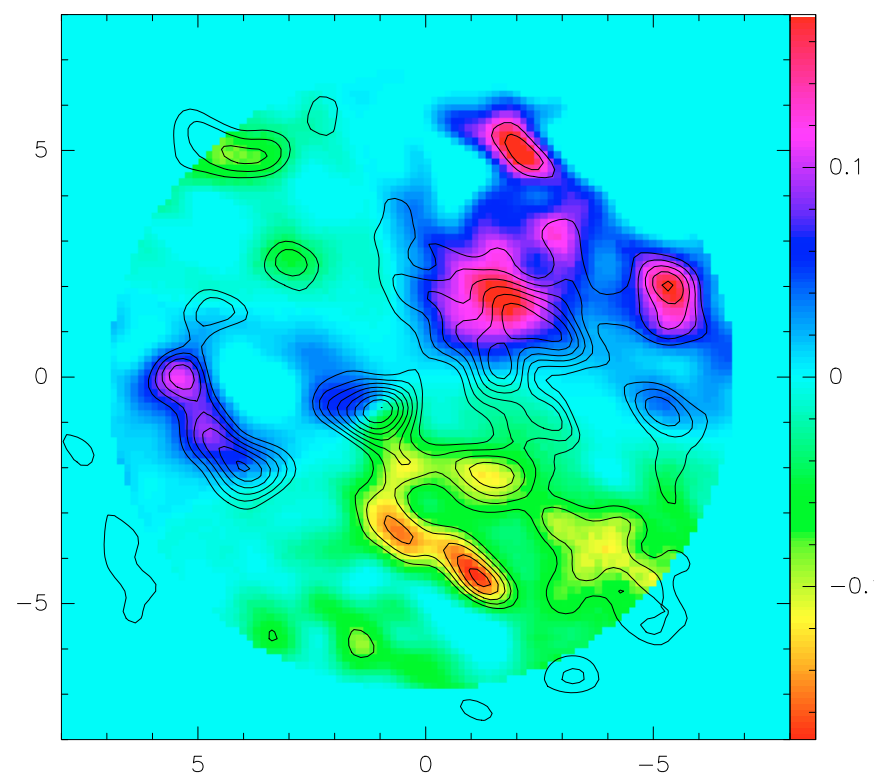

Fig. 22. Same as Fig. 21 for the ${ }^{12} \mathrm{CO}(2-1)$ emission taken as tracer of gas surface density.

broad-band optical image of NGC 5953 shows a flocculent spiral structure and an "S" like-bar of $\sim 250 \mathrm{pc}$ and $\gtrsim 60 \mathrm{pc}$ in size, respectively. These structures are absent in the $1.6 \mu \mathrm{m}$ (HST/NICMOS/F160W) image and in the IRAC $8 \mu \mathrm{m}$ "dustonly" one; instead in the optical and at $1.6 \mu \mathrm{m}$ we identified at $\sim 3^{\prime \prime}$ west of center the foreground star studied by Rafanelli et al. (1990). The stellar structure has been investigated by performing a two-dimensional B/D decomposition on the ground-based $H$-band image and IRAC $3.6 \mu \mathrm{m}$ image. Both sets of residuals show a circumnuclear stellar ring with a radius of $\sim 5-7^{\prime \prime}$, approximately coincident in size with the ${ }^{12} \mathrm{CO}$ disk. The presence of the ring in both sets of residuals suggests that it is not the typical "doughnut" associated with the diffraction limit and IRAC PRF incompatibilities, but rather is a real feature. The size of this stellar ring is the same of the circumnuclear star-forming ring seen in $\mathrm{H} \alpha$ with previous observations. In addition, since this ring is visible in the NIR as stars but not as hot dust emission $(J-K)$, we interpret it as a RSG population at least 10 Myr old.

There is an apparent counter-rotation between gas and stars inside the ring, and stars outside. The ring could be the separation between the two kinematically decoupled components (KDC). The formation of a KDC could be explained as a result of the ongoing interaction, and could be in its early stages. Alternatively, this apparent counter-rotation could be due to a warp of the plane of the disk. The ring would then be the start of the warp.

Using NIR images we found that gravity torques acting on the gas are predominantly positive in the region $\sim 100-400 \mathrm{pc}$ in both ${ }^{12} \mathrm{CO}(1-0)$ and ${ }^{12} \mathrm{CO}(2-1)$, indicating that the gas is not fueling the central $\sim 100 \mathrm{pc}$ region, down to the effective spatial resolution of our observations. In addition, the absolute values of these torques are very small, certainly due to the almost axisymmetric total mass and gas distributions in the center of the galaxy. The AGN in NGC 5953 is apparently not being actively fueled in the current epoch.

Acknowledgements. The authors would like to thank the anonymous referee, whose comments have been useful for improving the original version of the paper. We thank the scientific and technical staff at IRAM for their work in making our $30 \mathrm{~m}$ and PdBI observations possible. V. Casasola wishes to thank 

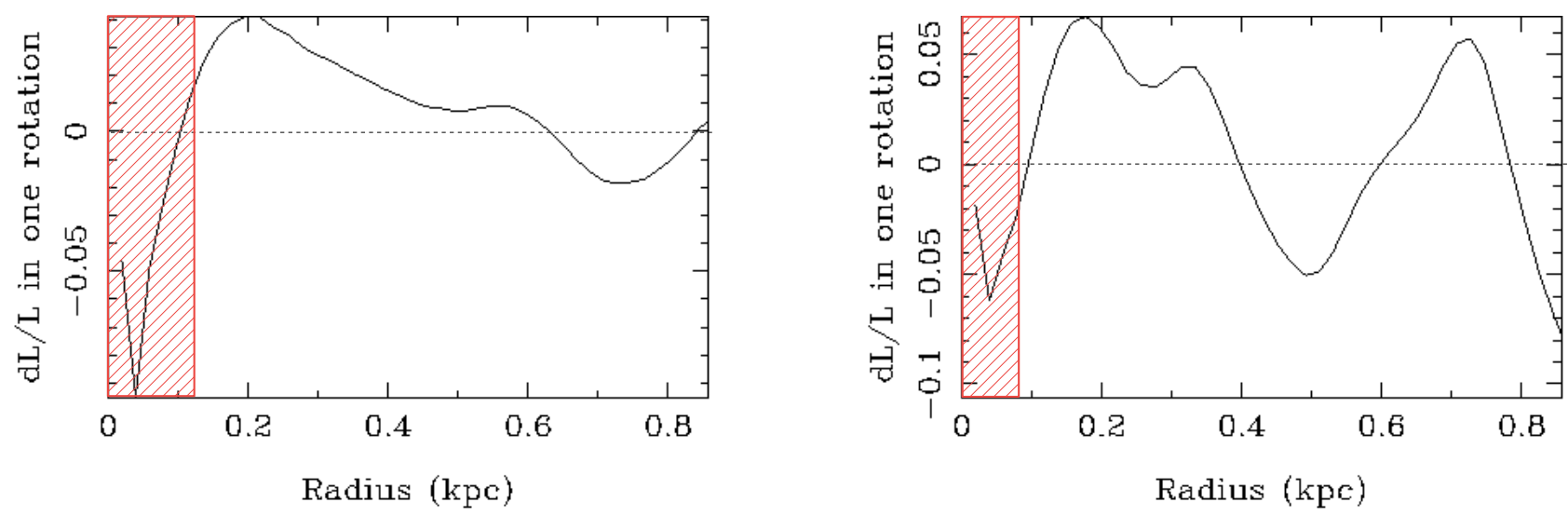

Fig. 23. The torque, or more precisely the fraction of the angular momentum transferred from/to the gas in one rotation $-d L / L-i s$ plotted for ${ }^{12} \mathrm{CO}(1-0)($ left $)$ and ${ }^{12} \mathrm{CO}(2-1)(r i g h t)$. The (red) dashed area corresponds to the resolution limit of our observations. In the left panel, the $\mathrm{CO}(1-0)$ map resolution is the limiting factor, while, on the right, the $\mathrm{CO}(2-1)$ and the similar NIR image resolution are the constraining factors.

Riccardo Cesaroni and Claudio Codella for help provided during the data reduction. This research has made use of the NASA/IPAC Extragalactic Database (NED), HyperLeda Database, IRAS Catalog, Spitzer archive, and Hubble Legacy Archive.

\section{References}

Bigiel, F., Leroy, A., Walter, F., et al. 2008, AJ, 136, 2846

Boone, F., Baker, A. J., Schinnerer, E., 2007, A\&A, 471, 113 (NUGA VII)

Boselli, A., Lequeux, J., \& Gavazzi, G. 2002, Ap\&SS, 281, 127

Braine, J., \& Combes, F. 1992, A\&A, 264, 433

Cardelli, J. A., Clayton, G. C., \& Mathis, J. S. 1989, ApJ, 345, 245

Casasola, V., Bettoni, D., \& Galletta, G. 2004, A\&A, 422, 941

Casasola, V., Combes, F., Bettoni, D., et al. 2007, A\&A, 473, 771

Casasola, V., Combes, F., García-Burillo, S., et al. 2008, A\&A, 490, 61

Chengalur, J. N., Salpeter, E. E., \& Terzian, Y. 1994, AJ, 107, 1984

Combes, F. 2001, Advanced Lectures on the Starburst-AGN, 223

Combes, F., \& Sanders, R. H. 1981, A\&A, 96, 164

Combes, F., \& Gerin, M. 1985, A\&A, 150, 327

Combes, F., García-Burillo, S., Boone, F., et al. 2004, A\&A, 414, 857 (NUGA II)

Combes, F., Baker, A. J., Schinnerer, E., et al. 2009, A\&A, 503, 73 (NUGA XII)

di Matteo, P., Combes, F., Melchior, A.-L., et al. 2008, A\&A, 477, 437

Falcón-Barroso, J., Bacon, R., Bureau, M., et al. 2006, MNRAS, 369, 529

Ferrarese, L., Pogge, R. W., Peterson, B. M., et al. 2001, ApJ, 555, L79

Friedli, D., \& Martinet, L. 1993, A\&A, 277, 27

Gao, Y., \& Solomon, P. M. 2004a, ApJ, 606, 271

Gao, Y., \& Solomon, P. M. 2004b, ApJS, 152, 63

García-Burillo, S., Guelin, M., \& Cernicharo, J. 1993, A\&A, 274, 123

García-Burillo, S., Sempere, M. J., Combes, F., Hunt, L. K., \& Neri, R. 2000, A\&A, 363, 869

García-Burillo, S., Combes, F., Hunt, L. K., et al. 2003, A\&A, 407, 485 (NUGA I)

García-Burillo, S., Combes, F., Schinnerer, E., Boone, F., \& Hunt, L. K. 2005, A\&A, 441, 1011 (NUGA IV)

García-Burillo, S., Fernández-García, S., Combes, F., et al. 2009, A\&A, 496, 85 (NUGA XI)

Graciá-Carpio, J., García-Burillo, S., Planesas, P., Fuente, A., \& Usero, A. 2008, A\&A, 479, 703

Gonzalez Delgado, R. M., \& Perez, E. 1996, MNRAS, 281, 781

Guilloteau, S., \& Lucas, R. 2000, ASP Conf. Proc. 217, Imaging at Radio through Submillimeter Wavelengths, 299

Haan, S., Schinnerer, E., Mundell, C. G., et al. 2007, Astron. Nachr., 328, 675

Haan, S., Schinnerer, E., Mundell, C. G., García-Burillo, S., \& Combes, F. 2008, AJ, 135, 232

Heckman, T. M., Smith, E. P., Baum, S. A., et al. 1986, ApJ, 311, 526

Heckman, T. M., Kauffmann, G., Brinchmann, J., 2004, ApJ, 613, 109

Helou, G., Roussel, H., Appleton, P., et al. 2004, ApJS, 154, 253

Hernández-Toledo, H. M., Fuentes-Carrera, I., Rosado, M., et al. 2003, A\&A, 412,669

Hibbard, J. E., \& van Gorkom, J. H. 1996, AJ, 111, 655

Hopkins, P. F., \& Hernquist, L. 2006, ApJS, 166, 1

Hunt, L. K., \& Malkan, M. A. 2004, ApJ, 616, 707
Hunt, L. K., Malkan, M. A., Rush, B., et al. 1999, ApJS, 125, 349 Hunt, L. K., Pierini, D., \& Giovanardi, C. 2004, A\&A, 414, 905

Hunt, L. K., Combes, F., García-Burillo, S., et al. 2008, A\&A, 482, 133 (NUGA IX)

Iono, D., Yun, M. S., \& Ho, P. T. P. 2005, ApJS, 158, 1

Janiuk, A., Czerny, B., Siemiginowska, A., et al. 2004, ApJ, 602, 595

Jenkins, C. R. 1984, ApJ, 277, 501

Jogee, S., Scoville, N., \& Kenney, J. D. P. 2005, ApJ, 630, 837

Jogee, S. 2006, Physics of Active Galactic Nuclei at all Scales, Lect. Notes Phys.,

693, 143

Kenney, J. D., \& Young, J. S. 1986, ApJ, 301, L13

Kennicutt, R. C., Jr. 1998, ARA\&A, 36, 189

King, A. R., \& Pringle, J. E. 2007, MNRAS, 377, L25

Knapen, J. H., Pérez-Ramírez, D., \& Laine, S. 2002, MNRAS, 337, 808

Kohno, K., Ishizuki, S., Matsushita, S., Vila-Vilaro, B., \& Kawabe, R. 2003, PASJ, 55, L1

Krips, M., Eckart, A., \& Neri, R. 2005, A\&A, 442, 479 (NUGA III)

Krips, M., Eckart, A., Krichbaum, T. P., et al. 2007a, A\&A, 464, 553

Krips, M., Neri R., García-Burillo, S. 2007b, A\&A, 468, L63, (NUGA VI)

Leitherer, C., Schaerer, D., Goldader, J. D., et al. 1999, ApJS, 123, 3

Leroy, A. K., Walter, F., Brinks, E., et al. 2008, AJ, 136, 2782

Makovoz, D., \& Marleau, F. R. 2005, PASP, 117, 1113

Malkan, M. A., Gorjian, V., \& Tam, R. 1998, ApJS, 117, 25

Maloney, P., \& Black, J. H. 1988, ApJ, 325, 389

Marecki, A., Spencer, R. E., \& Kunert, M. 2003, Publ. Astron. Soc. Austr., IAU Symp., 20, 46

Moriondo, G., Giovanardi, C., \& Hunt, L. K. 1998, A\&AS, 130, 81

Mulchaey, J. S., \& Regan, M. W. 1997, ApJ, 482, L135

Nakai, N., \& Kuno, N. 1995, PASJ, 47, 761

Narayanan, D., Cox, Th. J., Robertson, B., et al. 2006, ApJ, 642, L107

Paturel, G., Petit, C., Prugniel, P., et al. 2003, A\&A, 412, 45

Peng, C. Y., Ho, L. C., Impey, C. D., et al. 2002, AJ, 124, 266

Rafanelli, P., Osterbrock, D. E., \& Pogge, R. W. 1990, AJ, 99, 53

Rampazzo, R., Reduzzi, L., Sulentic, J. W., et al. 1995, A\&AS, 110, 131

Regan, M. W., \& Mulchaey, J. S. 1999, AJ, 117, 2676

Sakamoto, K., Okumura, S. K., Ishizuki, S., et al. 1999, ApJ, 525, 691

Schinnerer, E., Eckart, A., \& Tacconi, L. J. 2000a, ApJ, 533, 826

Schinnerer, E., Eckart, A., Tacconi, L. J., Genzel, R., \& Downes, D. 2000b, ApJ, 533,850

Shu, F. H., Tremaine, S., Adams, F. C., et al. 1990, ApJ, 358, 495

Solomon, P. M., \& Barrett, J. W. 1991, Dynamics of Galaxies and Their Molecular Cloud Distributions, 146, 235

Veilleux, S., Kim, D.-C., Sanders, D. B., Mazzarella, J. M., \& Soifer, B. T. 1995, ApJS, 98, 171

Vollmer, B., Braine, J., Balkowski, C., Cayatte, V., \& Duschl, W. J. 2001, A\&A, 374,824

Wilson, C. D. 1995, ApJ, 448, L97

Wu, J., Evans, N. J., II, Gao, Y., et al. 2005, ApJ, 635, L173

Yao, L., Seaquist, E. R., Kuno, N., et al. 2003, ApJ, 588, 771

Young, J. S., Xie, S., Tacconi, L., et al. 1995, ApJS, 98, 219

Zhu, M., Seaquist, E. R., Davoust, E., Frayer, D. T., \& Bushouse, H. A. 1999, AJ, 118, 145 